\title{
Multidimensional Waveform Encoding: A New Digital Beamforming Technique for Synthetic Aperture Radar Remote Sensing
}

\author{
Gerhard Krieger, Member, IEEE, Nicolas Gebert, and Alberto Moreira, Fellow, IEEE
}

\begin{abstract}
This paper introduces the innovative concept of multidimensional waveform encoding for spaceborne synthetic aperture radar (SAR). The combination of this technique with digital beamforming on receive enables a new generation of SAR systems with improved performance and flexible imaging capabilities. Examples are high-resolution wide-swath radar imaging with compact antennas, enhanced sensitivity for applications like alongtrack interferometry and moving object indication, and the implementation of hybrid SAR imaging modes that are well suited to satisfy hitherto incompatible user requirements. Implementationspecific issues are discussed and performance examples demonstrate the potential of the new technique for different remote sensing applications.
\end{abstract}

Index Terms-Adaptive radar, along-track interferometry (ATI), ambiguity reduction, digital beamforming, displaced phase centre antenna (DPCA), ground moving target indication (GMTI), high-resolution wide-swath SAR imaging, hybrid SAR, MIMO SAR, synthetic aperture radar (SAR), waveform diversity.

\section{INTRODUCTION}

W IDE UNAMBIGUOUS swath coverage and high azimuth resolution pose contradicting requirements on the design of spaceborne synthetic aperture radar (SAR) systems [1]-[4]. This motivated the development of advanced SAR imaging modes with different tradeoffs between spatial coverage and azimuth resolution. The classical solution to wide-swath SAR imaging is ScanSAR, which increases the unambiguous swath width at the cost of an impaired azimuth resolution [5], [6]. The ScanSAR mode is of high importance for all spaceborne remote sensing applications that require frequent monitoring of large areas. Examples are regular land use and vegetation mapping, marine observation, and monitoring of the Arctic sea ice. Another prominent example is differential interferometry, which compares the phase of complex SAR images acquired during different satellite passes to detect subtle deformations on the Earth's surface for many geoscience and environmental applications [7], [8]. All these applications ask for short revisit times. This implies, in turn, that a wide swath must be imaged to ensure seamless coverage with a single

Manuscript received November 10, 2006; revised June 1, 2007.

The authors are with the Microwaves and Radar Institute (HR), German Aerospace Center (DLR), 82234 Wessling, Germany (e-mail: gerhard.krieger@ dlr.de).

Color versions of one or more of the figures in this paper are available online at http://ieeexplore.ieee.org.

Digital Object Identifier 10.1109/TGRS.2007.905974 satellite. Wide-swath SAR imaging is also a key requirement for the design of the forthcoming Sentinel-1 satellite mission, which will employ an optimized ScanSAR technique to obtain frequent observations of large areas on the Earth's surface [9], [10].

On the other hand, there exist many remote sensing applications that ask for SAR images with a high spatial resolution. Examples are marine and terrestrial surveillance, cartographic mapping, monitoring of urban areas, as well as hazard and damage assessment. Even repeat-pass interferometry would benefit from a refined resolution. The rising demand for finely resolved SAR images led to the development of the spotlight mode, which improves the azimuth resolution at the cost of noncontiguous coverage along the satellite track [11]. The spotlight technique is also adopted in current satellite missions like TerraSAR-X, Radarsat-2, COSMO SkyMed, and SAR-Lupe to acquire SAR images with spatial resolutions in the meter or even decimeter range [12]-[15]. Up to now, it is, however, not possible to combine the benefits from spotlight and ScanSAR imaging in one and the same data take. This dilemma motivated research toward the development of new radar techniques that allow for the acquisition of spaceborne high-resolution SAR imagery without the classical coverage limitations imposed by range and azimuth ambiguities.

A promising candidate for such a new radar technique is digital beamforming on receive, where the receiving antenna is split into multiple subapertures. In contrast to analog beamforming, the received signals of each subaperture element are separately amplified, down-converted, and digitized, as shown in Fig. 1. This enables an a posteriori combination of the recorded subaperture signals to form multiple beams with adaptive shapes. The additional information about the direction of the scattered radar echoes can then be used to: 1) suppress spatially ambiguous signal returns from the ground; 2) increase the receiving antenna gain without a reduction of the imaged area; 3 ) suppress spatially localized interferences; and 4) gain additional information about the dynamic behavior of the scatterers and their surroundings. By this, it becomes possible to overcome fundamental limitations of conventional SAR systems. Several proposals have been made to use digital beamforming on receive for the development of SAR imaging systems with wide swath coverage and high azimuth resolution [16]-[34]. All these proposals employ a small fixed aperture antenna to illuminate a wide swath on the ground. The scattered radar echoes are then recorded by a large antenna array, which 


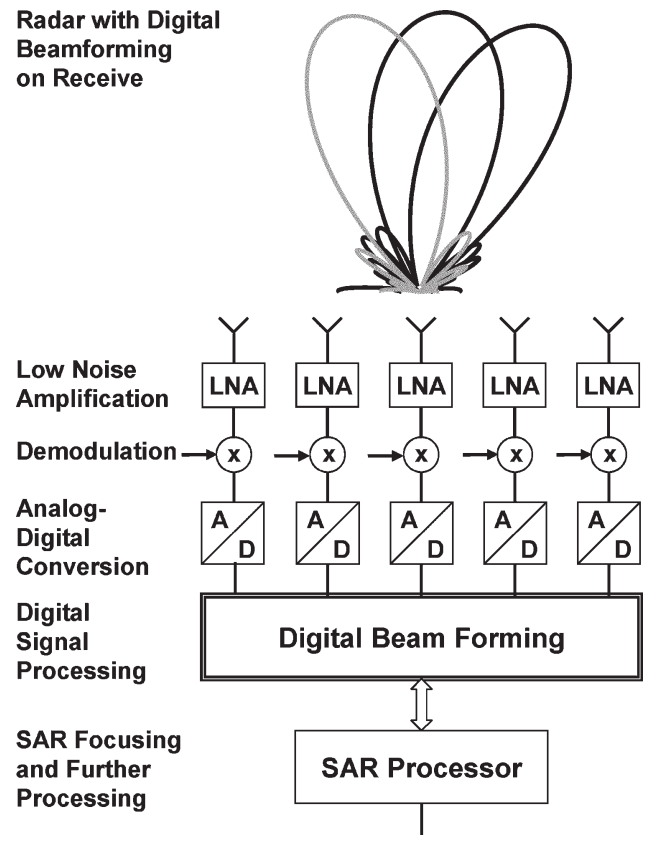

Fig. 1. Schematic of digital beamforming on receive. The signal from each subaperture element is independently amplified, down-converted, and digitized. The digital processing enables flexible and adaptive beamforming after signal reception.

enables the suppression of SAR ambiguities and compensates the gain loss that arises from a wide-area illumination.

This paper presents a novel fully active digital beamforming technique for radar remote sensing that is based on the concept of multidimensional waveform encoding on transmit [35], [36]. In contrast to the aforementioned techniques, the full area of the antenna aperture is used for both the transmission and the reception of radar pulses. This enables an improved SAR imaging performance while relying on the well-established transmit/receive (T/R) module technology. The mandatory expansion of the antenna beam for a wide-area illumination is achieved by an innovative spatiotemporal modulation of each transmitted radar pulse. The modulation introduces angular waveform diversity in the transmitted signal and provides, thereby, in the recorded radar echoes additional information about the spatial scatterer distribution. The multidimensional waveform encoding is hence the natural complement to digital beamforming on receive, and the combination of both techniques enables a wealth of new SAR imaging modes that significantly increase the performance, flexibility, and adaptability of future radar systems and missions. This will, in turn, pave the way for a new era in spaceborne radar remote sensing, serving an increasing range of powerful applications.

This paper is organized as follows. Section II reviews the signal reception in a multiple-aperture SAR from an informationtheoretic point of view. In this context, inherent challenges of multichannel SAR systems are discussed, and we also give first hints for possible solutions. Section III then introduces the innovative concept of multidimensional radar waveform encoding on transmit. The great potentials of this new technique for the development of future SAR systems are demonstrated in Sections IV and V, using intrapulse beamsteering in elevation and multidimensional waveform encoding in azimuth as illus- trative examples. Section VI gives hints for system optimization and discusses advanced opportunities like the implementation of hybrid SAR modes. This paper concludes with a short summary in Section VII.

\section{Multiaperture Signal Reception}

\section{A. High-Resolution Wide-Swath SAR Imaging}

Several suggestions have been made in the open literature to employ a multichannel receiver system for high-resolution wide-swath SAR imaging [16]-[34]. All of them use either a small portion of a large $\mathrm{Tx} / \mathrm{Rx}$ antenna array or a separate transmit aperture to illuminate a large footprint on the ground, as shown in the schematic drawings of Fig. 2. The scattered signal is then received by multiple independent subaperture elements that are connected to individual recording channels. The combination of the subaperture signals enables the formation of an arbitrary number of narrow Rx beams a posteriori to the digital signal recording. The footprints of some exemplary $\mathrm{Rx}$ beams are shown in Fig. 2 as red dotted circles and ellipses.

The drawing in the left column of Fig. 2 illustrates the basic idea of a multiple beam SAR design, employing a squinted imaging geometry as suggested in [16] and [18]. In contrast to a conventional side-looking SAR, the antenna is rotated about its vertical axis and uses multiple horizontally displaced aperture elements with increased vertical extension for signal reception. Such an antenna arrangement enables the formation of multiple narrow beams that limit the Rx antenna footprints in both the along-track and cross-track directions to a small area on the Earth's surface. This allows, in turn, for the unambiguous SAR imaging of narrow swaths, and by combining the images from multiple Rx beams, one will be able to map a wide swath at nearly constant incident angle. Note that the echoes from all swaths arrive at approximately the same time, and hence, one may employ either a rather high pulse repetition frequency (PRF) and/or a long duty cycle without the risk for range ambiguities notwithstanding the wide image swath. A drawback of this solution is impaired spatial resolution and performance associated with high squint angles. Further problems arise for the SAR processing due to the large range walk that results from the linear component of the range cell migration.

Another approach to high-resolution wide-swath SAR imaging is the displaced phase center antenna (DPCA) technique [20]. The basic idea behind the DPCA concept is to acquire for each transmitted radar pulse additional raw data samples along the synthetic aperture. This may be achieved by employing a multichannel receiver in combination with multiple aperture elements that are mutually displaced in the along-track direction, as shown in the second column of Fig. 2. Consequently, some of the "synthetic" aperture samples are replaced by "real" aperture samples, which allows for a reduction of the required PRF without rising azimuth ambiguities. ${ }^{1}$ The DPCA technique, hence, takes full advantage of the total antenna length for azimuth ambiguity suppression. The azimuth resolution is, however, no longer determined by the length of the whole antenna array but

\footnotetext{
${ }^{1}$ This opportunity for azimuth ambiguity suppression has already been demonstrated with airborne SAR data [37], [38].
} 


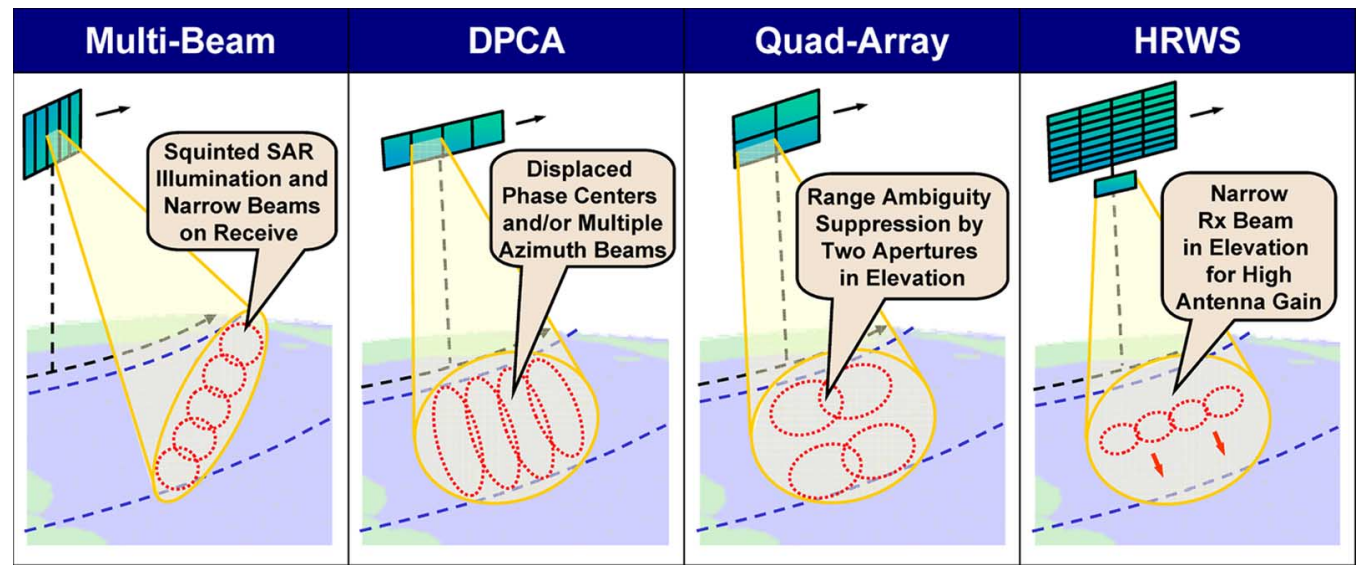

Fig. 2. Solutions for high-resolution wide-swath SAR imaging. (Left) Multiple beam SAR [16], [18]. (Middle left) DPCA technique [20]. (Middle right) Quadelement rectangular array SAR [21]. (Right) HRWS SAR [23], [25].

by the length of the individual subaperture elements. By this, it becomes possible to decouple the otherwise contradicting requirements for a high azimuth resolution and wide-swath SAR coverage. Note, however, that the DPCA technique by itself does, for a fixed total antenna length, not allow for an increase of the unambiguous swath width, but it is only well suited to improve the azimuth resolution.

An extension of the DPCA principle is the quad-element rectangular array system shown in the third column of Fig. 2. This system employs, in addition to the displaced azimuth apertures, multiple aperture rows in elevation [21]. The additional apertures in elevation enable now a reduction of the total antenna length since it becomes possible to increase for a fixed swath width the PRF and to suppress the resulting range ambiguities by an appropriate null steering in elevation [19]. An inherent disadvantage of this technique is, however, that the resulting swath is no longer contiguous since the receiver must be switched off during transmission. This leads to blind ranges and, therefore, gaps in the cross-track direction of the acquired scene. ${ }^{2}$

A further extension of the DPCA technique is the HRWS SAR system, which is shown in the right column of Fig. 2. This system combines a separate transmit antenna with a large receiver array [23], [25], [28], [32]. The small transmit antenna illuminates a wide swath on the ground, and the large receiver aperture compensates the Tx gain loss by a real-time digital beamforming process called scanning on receive. For this purpose, each azimuth panel of the receiving antenna is again divided into multiple vertically arranged subapertures. The signals from the individual subapertures are then combined in real time to form a narrow elevation beam that follows the radar pulse as it travels on the ground. By this, one may collect the radar echoes from a wide image swath despite using a receiver aperture with large vertical extension. The HRWS architecture is hence well suited to compensate the Tx gain loss from a wide area illuminator. The HRWS system concept

\footnotetext{
${ }^{2}$ Such a restriction could be overcome in a bistatic radar system where the spatial separation between the platforms allows for simultaneous transmission and reception [27]. Other opportunities to fill in the blind ranges are burst mode operation with different PRFs or even a continuous variation of the PRF.
}

and its implementation have been further investigated in several dedicated studies [28]-[33].

The following two sections discuss some general challenges and possible solutions related to the design and implementation of multichannel SAR systems. Section III will then build up on this discussion, and we will show how multidimensional waveform encoding can significantly improve the performance and flexibility of such systems.

\section{B. Multichannel SAR Processing}

The application of the DPCA technique for high-resolution wide-swath SAR imaging puts a stringent requirement on the PRF: the PRF has to be chosen such that the SAR platform moves just one half of the total antenna length between subsequent radar pulses [20]. This requirement is due to the fact that the effective phase centers of the additional samples from the multiaperture antenna must exactly fit in between the satellite's traveled distance between two transmit pulses to yield a uniformly sampled synthetic aperture. However, such a rigid selection of the PRF may be in conflict with the timing diagram; furthermore, it will exclude the opportunity to increase the PRF for improved azimuth ambiguity suppression. To avoid such restrictions, a new reconstruction algorithm has been developed in [30], which allows for the recovery of the unambiguous Doppler spectrum even in case of nonuniform DPCA sampling. The derivation of this reconstruction algorithm is based on modeling the raw data acquisition in a multiaperture SAR by a linear multichannel system model where each channel describes the data acquisition of one antenna element. The reconstruction assumes a limitation of the Doppler bandwidth $B_{\text {Dop }} \leq N_{\mathrm{Rx}} \cdot \mathrm{PRF}$, where $N_{\mathrm{Rx}}$ is the number of displaced receiver apertures in the along-track direction. A violation of this condition causes ambiguous returns as detailed in [33]. Such residual ambiguities can be avoided by an improved limitation of the azimuth spectrum prior to the signal recording. For example, beamforming on transmit can be used to obtain a refined azimuth antenna pattern with lower sidelobes and, therefore, a better limitation of the Doppler spectrum. Another possibility is a preshaping of the receiver beams. Here, the basic idea is to modify the azimuth beams of each receiver 
channel by combining the signals from overlapping subgroups of multiple receiving antenna elements prior to A/D conversion and storage. This, again, enables better sidelobe suppression and further allows a dynamic adaptation of the position of the effective phase centers, thereby improving the signal reconstruction [33]. In principle, one could also increase the number of azimuth channels while keeping the total antenna length fixed. This raises the number of samples of the synthetic aperture and, hence, enables more efficient suppression of residual ambiguities. A drawback of this solution is, of course, the increased data rate to be stored on the satellite and to be transmitted to the ground. The data volume can, however, be reduced onboard the satellite by a multichannel data compression that exploits the mutual redundancies (here, mainly second-order cross correlations) between the signals recorded from neighboring antenna elements. Optimum data compression algorithms and architectures can be derived from information theory by employing a rate distortion analysis [39], which has to take into account the desired performance of the final image product. This can be regarded as the more general view of the previously introduced preshaping of the receiver beams, and it will ultimately lead to the design of digital radar architectures, which are significantly driven by elements from information theory. ${ }^{3}$

\section{Information Content and Redundancy Reduction}

An independent recording of the signals from a large antenna array with multiple subaperture elements results in a huge amount of data samples, which exceeds, in general, by far the number of independent pixels in the final image. The recorded signals are hence redundant, and their mutual information can be regarded as being composed of two components. For this, we consider the illustration in Fig. 3, which shows a multiaperture antenna and the ground surface in the elevation plane. ${ }^{4}$ The first redundant component arises from the short duration of the transmitted radar pulse, which limits at each instance of time the extension of the scattering field on the ground. The restricted scattering area causes at each moment a strong spatial correlation among the signals from the different antenna elements. The second redundant component is an additional temporal correlation, which can be understood if we imagine the formation of a narrow Rx beam that covers only a part of the instantaneous scattering field. Assuming now a transmitted

\footnotetext{
${ }^{3}$ The data reduction by onboard preprocessing of the recorded azimuth signals could even be of interest for standard single-channel SAR systems. As a simple example, consider the standard stripmap mode in TerraSAR-X, where the final product is processed with a Doppler bandwidth of $2266 \mathrm{~Hz}$, while the employed PRF has typical values on the order of $3500 \mathrm{~Hz}$. Simple bandpass filtering and resampling of the recorded data before final storage and data transmission to the ground could, hence, reduce potential bottlenecks in both the onboard memory and the downlink capacity. This technique implicitly assumes total zero Doppler steering of the satellite [40] or at least an onboard estimation of the actual Doppler centroid. The onboard data reduction could, in principle, also be performed off-line after initial uncompressed raw data recording in an appropriate cache segment of the main memory. This reduces the demands for real-time processing, since it is now possible to use the idle times of the SAR instrument.

${ }^{4}$ For convenience, we will restrict the discussion in this section to multi aperture sampling in elevation, but most of the presented solutions can also be applied to azimuth, as indicated in Section II-B.
}

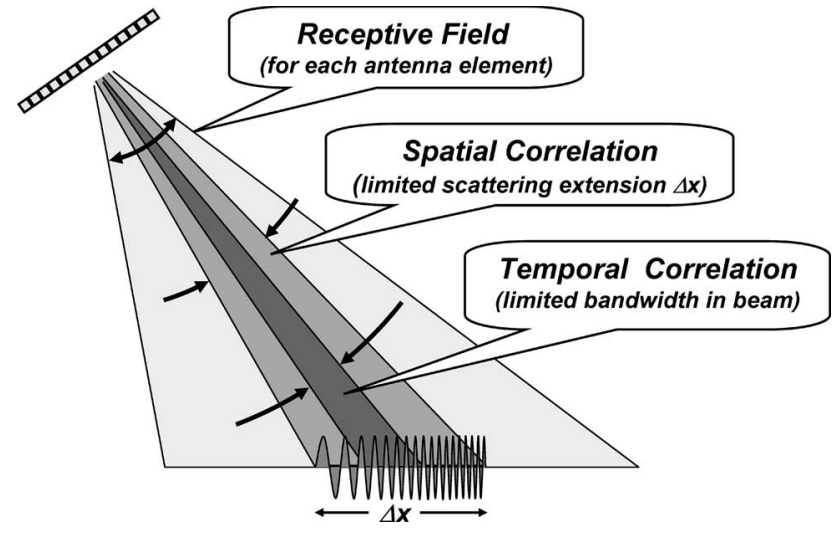

Fig. 3. Redundancy of recorded data.

radar pulse with a linear frequency modulation, only a part of the total range bandwidth will be visible at each instance of time, thereby introducing a temporal correlation among the samples of the recorded signal [27].

The temporal correlation can, in principle, be exploited for data compression by subsampling the signals from each subaperture element of the antenna array, as shown on the lower left part of Fig. 5. This is because the scattered signal arrives at each antenna element with a different range delay. By this, one obtains additional samples of the RF signal, and for large antenna arrays, one can recover the unambiguous $\mathrm{RF}$ signal spectrum in analogy to the previously introduced multiple-aperture technique for azimuth ambiguity suppression. However, mere subsampling in either space or time would also increase the noise level due to noise aliasing, thereby loosing the opportunity for coherent averaging. A more efficient exploitation of the redundancies requires a spatiotemporal transformation of the recorded antenna signals, which projects the useful signal energy onto a compact subspace of the totally recorded data volume. An example for such a transformation is the real-time beamscanning in elevation, as suggested for the HRWS system in [23] and [25]. This technique combines the signals from all antenna elements to form a narrow beam, which follows the radar pulse as it travels on the ground. This is achieved by selecting the scan angle as a function of range time. ${ }^{5}$ The principle drawback of this deterministic and completely data independent coupling is that important information may irrecoverably be lost during data recording. For example, isorange elevation changes in mountainous terrains will cause a reduced antenna gain due to erroneous beam pointing, as shown in Fig. 4.

To quantify this effect, we consider a fully focused Rx beam, which is "pointed" to range $r(\tau)=r_{0}$. This range is associated with an incident angle $\theta_{\text {inc }}$ under the assumption of a flat terrain

\footnotetext{
${ }^{5}$ The common use of long Tx pulses in a spaceborne radar requires additional precautions since the instantaneous scattering field on the ground is typically wider than the footprint of the elevation beam (see also Fig. 3). The HRWS system solves this problem by assuming for the Tx signal a chirp with linear frequency modulation and making the scan angle also frequency dependent. This can easily be achieved for most systems by introducing additional time delays in the signal paths from the individual antenna elements prior to the final summation [25]. The following reasoning is completely independent of such additional precautions since it applies to all frequencies in the same manner.
} 


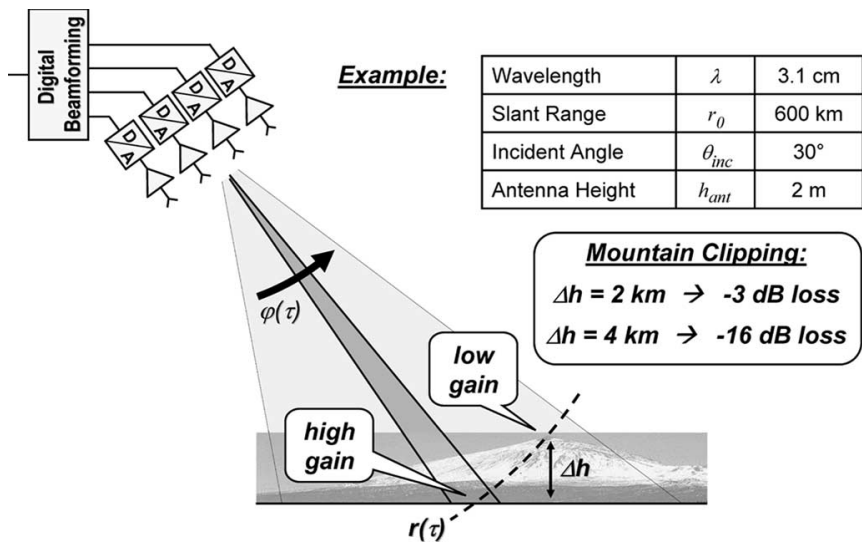

Fig. 4. Gain loss from topography during pulse chasing.

with height $h_{0}$. If the terrain height changes by a height of $\Delta h$ and the beam pointing remains the same, we will obtain a mispointing of the antenna beam by

$$
\Delta \varphi \approx \frac{\Delta h}{r_{0} \cdot \sin \left(\theta_{\mathrm{inc}}\right)} .
$$

Assuming now a receiver array that is oriented perpendicular to the line of sight, this will reduce the antenna gain by

$$
\Delta G_{\mathrm{Rx}}=\operatorname{si}\left(\frac{\pi \cdot h_{\mathrm{ant}}}{\lambda} \cdot \sin \left(\frac{\Delta h}{r_{0} \cdot \sin \left(\theta_{\mathrm{inc}}\right)}\right)\right)
$$

where $h_{\text {ant }}$ is the antenna height, $\lambda$ is the wavelength, and $\operatorname{si}(x)$ is the $\sin (x) / x$ function, which is used to approximate the directivity pattern of a rectangular antenna with a sufficiently dense subaperture spacing. The table on the right-hand side of Fig. 4 shows the expected gain loss for an X-band system with an antenna height of $2 \mathrm{~m}$ operating at a slant range of $600 \mathrm{~km}$ and an incident angle of $30^{\circ}$. The height differences of $\Delta h=2 \mathrm{~km}$ and $\Delta h=4 \mathrm{~km}$ amount to gain losses of -3 and $-16 \mathrm{~dB}$, respectively. This simple example shows that the effect of varying terrain heights cannot be neglected in the design of a spaceborne HRWS X-band system if the antenna height shall exceed 1-2 m. Antenna heights of $2 \mathrm{~m}$ and more are, however, desired to compensate the gain loss from a large area illuminator.

A possible solution to this problem is the simultaneous formation of multiple narrow beams and an adaptive selection of the data from the beam with maximum output power, as shown on the upper left part of Fig. 5. However, this approach still poses the problem of the possibility that there exist strong topography changes within the extension of the azimuth footprint. This will then lead to multiple beams that carry significant information about the imaged scene. An alternative is partial beamforming, which combines only a subset of the antenna elements, as shown on the upper right part of Fig. 5. The outputs of the beamformers are then combined by predictive coding and/or a joint (polar) quantization, which is well suited to exploit residual redundancies between the signals from the individual beams [41]. Optimized quantizers with a minimum data rate can be derived from a rate distortion analysis, and by employing a spatiotemporal multibeam vector quantizer, one
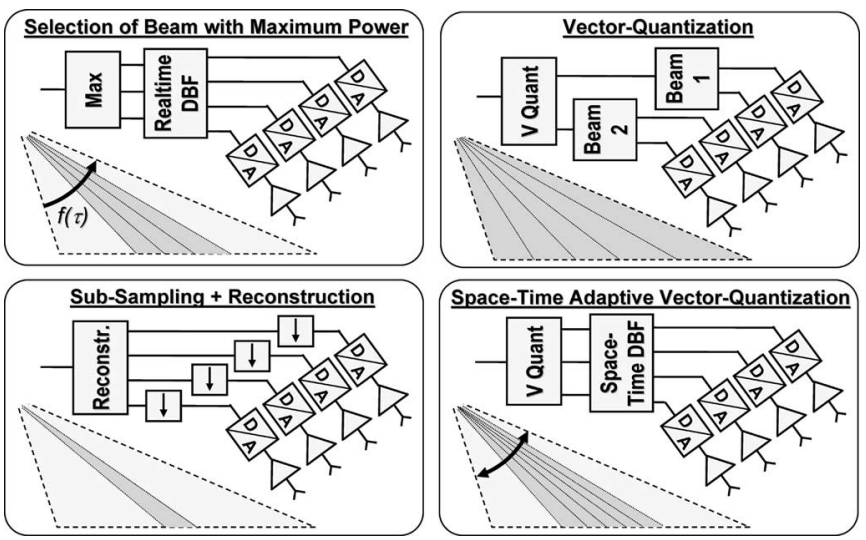

Fig. 5. Strategies for redundancy reduction in multiaperture SAR systems.

could even use a dynamic bit assignment with variable bit rates, which reflects the actual information content recorded by the large antenna array (Fig. 5, lower right). There exist, of course, further options for data reduction in multiaperture SAR systems, and we will come back to this fundamental issue from a completely different point of view in Section IV.

\section{Multidimensional WAVEForms}

\section{A. Limitations of a Fixed-Aperture Illumination}

The HRWS system concept introduced in Section II assumes a wide-area illumination by a separate transmit antenna. This enables an independent electrical design and optimization of the transmit and receive paths, but it also requires the accommodation of an additional antenna on the spacecraft and reduces the flexibility to operate the radar system in different SAR imaging modes like ultrawide-swath ScanSAR, high signal-tonoise ratio (SNR) spotlight, or new hybrid modes to be discussed later. Furthermore, the small Tx antenna aperture causes a finite roll-off of the antenna pattern and, hence, a nonoptimum RF power distribution within the illuminated footprint, which manifests itself as a performance degradation at the swath border. This gain-loss may, in turn, increase the necessary size of the total $\mathrm{Rx}$ aperture. A further challenge arises for the mapping of swaths with different incident angles, which requires either a roll maneuver of the whole satellite or a more complex Tx antenna design that involves, e.g., multiple feeds in case of employing reflector antenna technology [32]. Another peculiarity of the HRWS system is the fact that the required length of the Rx antenna is still proportional to the swath width, as in a conventional SAR system [27]. The mapping of a wide image swath, hence, requires a rather long Rx antenna, which complicates its accommodation on the spacecraft and increases the launch costs.

It is hence also worth considering the application of digital beamforming techniques in radar systems that use the same antenna array for both the transmission and reception of radar pulses, thereby taking advantage of the extended size of the $\mathrm{Rx}$ antenna array in combination with the already-existing $\mathrm{T} / \mathrm{R}$ module technology. The use of a large Tx aperture for highresolution wide-swath SAR imaging poses, in turn, the question of how the signal energy on the ground can be distributed. The 

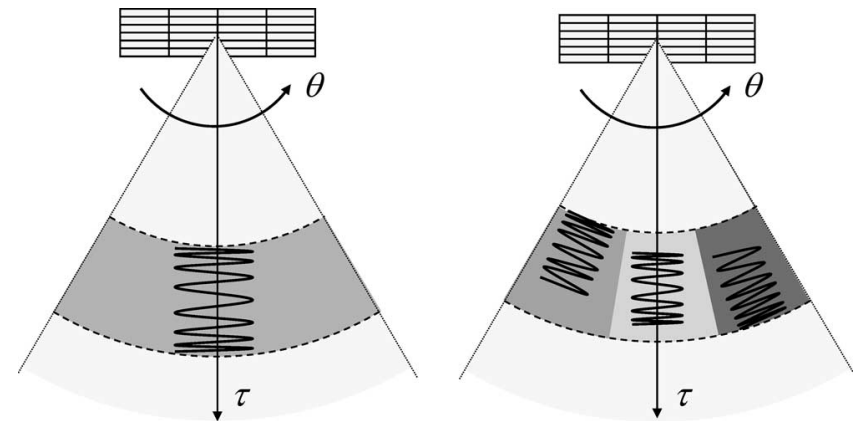

Fig. 6. Separable and nonseparable waveforms. (Left) Separable radar pulse, as used in all conventional SAR systems and imaging modes. (Right) Nonseparable waveform, allowing for multidimensional encoding of the transmitted radar pulse.

trivial solution would be amplitude tapering, or, as an extreme case, the use of only a part of the transmit antenna; but this causes a significant loss of radiated power; furthermore, it may lower the overall efficiency since the high-power amplifiers in the $\mathrm{T} / \mathrm{R}$ modules are no longer driven in saturation. Another solution is phase tapering, but the derivation of appropriate phase coefficients is a complex task that requires, in general, complicated numerical optimization techniques [42], [43].

\section{B. Nonseparable Radar Pulses}

A completely different approach to exploit the large antenna array for signal transmission is the employment of a spatiotemporally nonseparable waveform for each transmitted radar pulse. Such a waveform is characterized by the inequality

$$
w\left(\tau, \theta_{\mathrm{el}}, \theta_{\mathrm{az}}\right) \neq h(\tau) \cdot a\left(\theta_{\mathrm{el}}\right) \cdot b\left(\theta_{\mathrm{az}}\right)
$$

where $h(\tau)$ describes the temporal structure of the modulated RF radar pulse, $a\left(\theta_{\mathrm{el}}\right)$ describes the weighting from the antenna pattern in elevation, and $b\left(\theta_{\mathrm{az}}\right)$ describes the weighting from the antenna pattern in azimuth. The illustration in Fig. 6 visualizes the difference between a nonseparable waveform encoding (right) and a separable transmit pulse (left), as used up to now in all air- and spaceborne SAR systems and imaging modes.

One can imagine a multitude of solutions for the technical implementation of multidimensional waveforms in a multiaperture SAR system. These solutions substantially differ with regard to their degree of complexity. A very simple means to obtain a multidimensional waveform is the division of a long transmit pulse into multiple subpulses. Each subpulse is then associated with a different antenna beam via switching the phase coefficients in the T/R modules between successive subpulses. A completely different and more complex solution is the use of a separate waveform generator for each subaperture element. Fig. 7 shows a potential hardware implementation that combines both techniques in one and the same system by employing analog subpulse phase switching in elevation and digital waveform generation in azimuth.

As a first example, we now consider the generation of nonseparable waveforms in space and time by merely switching between different antenna beams and/or subaperture elements

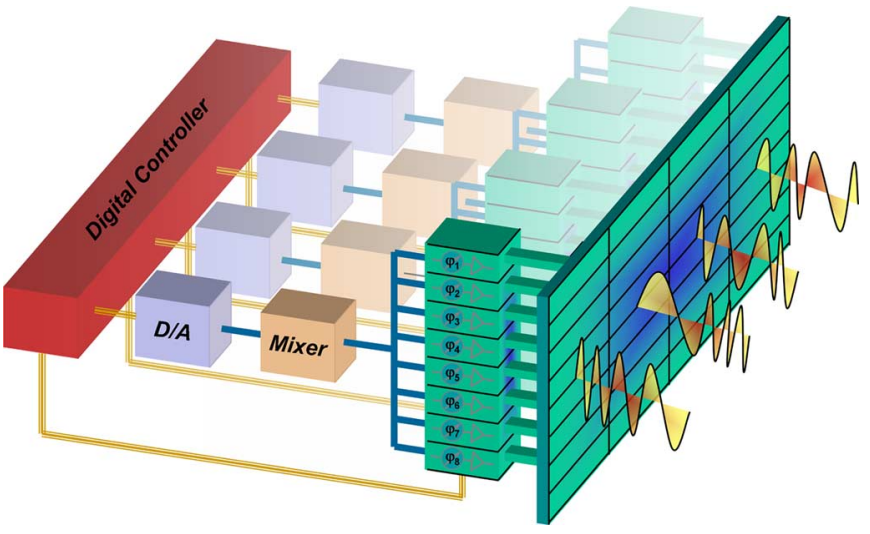

Fig. 7. Transmitter for multidimensional radar pulse encoding with four azimuth waveform generators and real-time intrapulse elevation beamsteering.

during each transmitted pulse. ${ }^{6}$ The overall PRF remains unaltered in this case. Since many of the previously mentioned applications rely on a rather low PRF on the order of $1-2 \mathrm{kHz}$, one may typically employ long pulse durations, and hence, we expect no technical problems for the concatenation of multiple subpulses with different antenna excitation patterns to a long pulse. We may even allow for subpulse switching times on the order of several microseconds without loosing too much of the duty cycle. A full-range resolution within each subbeam is achieved by concatenating multiple chirp signals in a sawtoothlike frequency modulation (or any other sequence of full bandwidth and possibly even mutually orthogonal waveforms). The advantage of such a scheme is that it allows the staggered illumination of a large area on the Earth's surface during each transmitted pulse, thereby supporting a highly flexible and adaptable distribution of the available signal energy within this area. This opportunity for a staggered wide-swath illumination with large antenna arrays will be investigated in Section IV. A further advantage arises for improved azimuth ambiguity suppression due to the reduced antenna beamwidth for each subpulse. This will be discussed together with other advantages in Section V.

The concept of multidimensional waveform encoding can, of course, be extended to an arbitrary spatiotemporal radar illumination, where each direction has its own temporal transmit signal with different power, duration, and phase code. Another opportunity is a systematic decomposition of the overall transmitted range frequency spectrum into multiple subbands. Each subband is then associated with a different subaperture and/or beam. Such a frequency decomposition of the transmitted range pulse may, again, be combined with intrapulse aperture switching and/or beamsteering in azimuth. This enables the illumination of a wide swath on the ground notwithstanding a

\footnotetext{
${ }^{6}$ Note that this should not be confused with the aperture switching technique introduced in [38] and [44], which toggles between different azimuth panels of the Tx antenna from a PRF pulse to another PRF pulse. This pulse-to-pulse aperture toggling is comparable to the ping-pong mode in SAR interferometry [45], [46], the synthetic bandwidth method [47], or the real aperture enlargement suggested for forward- and/or downward-looking radar systems [48], [49]. All these techniques interleave successive pulses by the receiving window and the spatiotemporal diversity, hence, requires an increase of the PRF to avoid azimuth ambiguities.
} 
large extension of the total Tx antenna array in elevation and the simultaneous suppression of azimuth ambiguities, as explained in Section V.

The selection of the spatiotemporal excitation coefficients in the transmitter could even be made adaptive by evaluating the recorded samples from previous radar echoes. By this, a closed loop will be formed between the radar sensor and its environment, which allows for maximization of the information that can be derived about the imaged scene for a given RF power budget. In analogy to the information-theoretic modeling of multiple-input multiple-output (MIMO) communication systems, such an optimization could then be regarded as maximizing the mutual information between the recorded radar signals and the scatterer distribution on the ground, thereby making optimum use of the channel capacity provided by a multiple antenna Tx/Rx radar system. As a simple example, one may consider the automatic compensation of angular differences in the received Rx signal power arising from variations in the length and attenuation of the propagation path and/or spatial inhomogeneities in the first-order scattering statistics of the imaged scene. The latter may be due to changing incident angles, land-water or water-ice transitions, as well as varying types of land cover. A spatiotemporal adaptation of the transmitted signal power is hence well suited to provide a more homogeneous image quality. The following sections provide further and more elaborate examples for the multitude of benefits and opportunities provided by spatiotemporal encoding of the transmitted radar pulses.

\section{INTRAPUlse BEAMSTEERING IN ELEVATION}

One example for multidimensional waveform encoding is intrapulse beamsteering in elevation. This enables an illumination of a wide image swath with a sequence of narrow and high-gain antenna beams. Such a staggered illumination is, in some sense, similar to the traditional ScanSAR mode, with the major difference that each transmitted pulse now illuminates not only one but all subswaths simultaneously. The illumination sequence within the Tx pulse can, in principle, be arranged in any order. An interesting opportunity arises if we start from a far-range illumination and subsequently proceed to the near range, as shown in Fig. 8. As a result, the radar echoes from different subswaths will overlap in the receiver, as shown on the upper right part of Fig. 8. The overall receiving window can, hence, be shortened, thereby reducing the amount of data to be recorded and stored on the satellite. In other words, the spatial data redundancy discussed in Section II is substantially reduced, since the receiver now obtains radar echoes from multiple directions simultaneously. This example illustrates the potential of multidimensional waveform encoding for data compression without sophisticated real-time onboard processing. The information space provided by multiple $\mathrm{Rx}$ apertures can moreover be filled by using sub-pulses with different Tx polarisations which allows for the implementation of a fully-polarimetric SAR without the necessity to increase the PRF by a factor of two. The temporal overlap of the radar echoes from the different subswaths and/or polarisations is then resolved in the spatial domain by digital beamform-

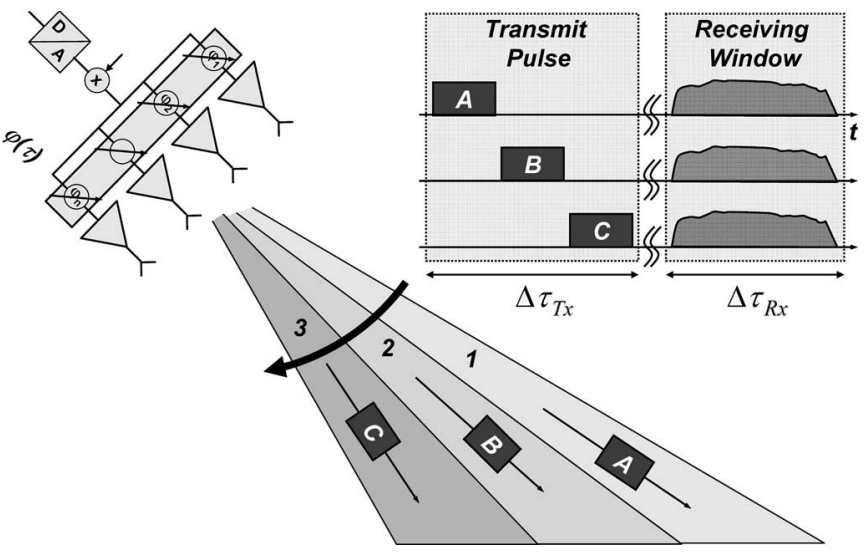

Fig. 8. Intrapulse beamsteering in elevation: backscattered signals from different subswaths superimpose in the receiving window.

ing on receive, where we exploit the relation between time delay and elevation angle in the sidelooking radar imaging geometry. This a posteriori processing can be performed on the ground, which has the further advantage that no information about the spatial structure of the recorded radar data will be lost, thereby enabling, e.g., suppression of directional interferences or jamming signals and avoiding the mountainclipping problem of real-time beam scanning discussed before (cf., Fig. 4).

A direct consequence of the short receiving window is the increased time available for the transmission of multiple subpulses. This reduces, in turn, the RF peak power requirements of the transmitter and gives further margin to switch between the subpulses, thereby simplifying the electrical system design. Another advantage is the reduced gain loss at the border of the swath if compared to a conventional radar illuminator. The staggered illumination allows even for a flexible distribution of the signal energy on the ground. As a simple example, one may consider the use of longer transmit pulses for subbeams with higher incident angles. This illumination strategy is well suited to compensate the SNR loss due to both the typical decrease of the backscattering with increasing incident angles and the additional free space loss from a larger range. As a result, one may reduce the overall power requirements of the radar payload, which, again, simplifies the thermal and electrical design of the satellite.

There exist several opportunities and tradeoffs to implement the required Tx beamsteering in elevation. One solution would be to use different (e.g., azimuth) parts of the large antenna array for each beam, thereby ensuring a low duty cycle for each T/R module notwithstanding the long transmit pulse. By this, one may also achieve the azimuth beam broadening, as desired for multiaperture high-resolution SAR imaging. In the limit, each T/R module needs only one preloaded phase coefficient, and the beamsteering is implemented by mere on/off switching between different subsets of T/R modules. Another solution is to use the full antenna array for the total pulselength, which has the advantage of further reducing the peak power requirements for each T/R module. The illumination is then achieved either by switching between multiple moderately phase tapered beams or even via continuous beamsteering from far to near range. 


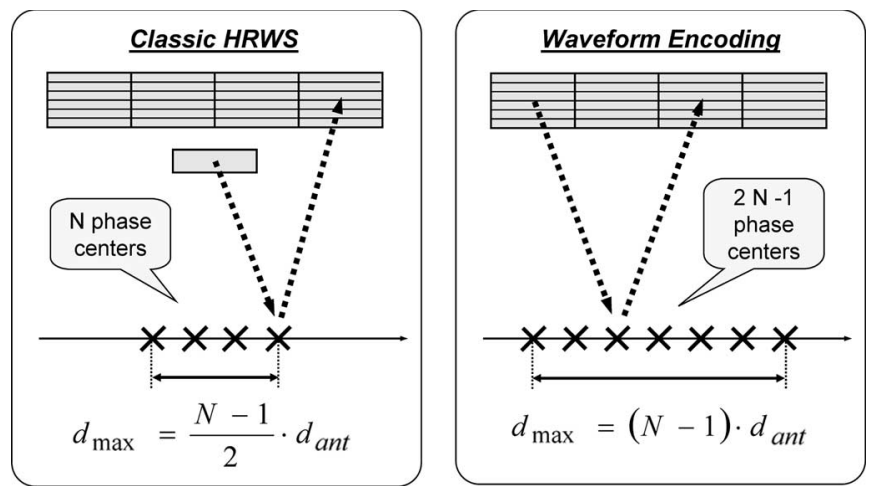

Fig. 9. Additional phase centers by nonseparable waveform encoding in azimuth.

The latter requires an appropriate temporal modulation of the transmitted RF signal such that each scatterer receives a sufficient bandwidth for high range resolution.

\section{Multidimensional WaVEForm ENCODING IN AZIMUTH}

\section{A. Waveform Diversity and Ambiguity Suppression}

Spatial modulation diversity in the radar transmitter can increase the information about the direction of a given scatterer. A simple example is a multiaperture antenna where each aperture transmits its own waveform, as shown in Fig. 7 for the azimuth direction. Possible waveforms are signals from different frequency subbands, time-shifted subpulses, or pulses with special phase codes that are similar to the up and down chirps suggested for range ambiguity suppression (cf., [50] and [51]). Orthogonal waveforms enable, in the case of isolated pointlike scatterers, a separation of the radar echoes from the different transmit signals, and the spatial diversity of the transmit phase centers causes relative phase shifts between the received waveforms for a given scatterer on the ground. This additional phase information can then be used to suppress azimuth ambiguities or to increase the sensitivity to object movements.

The performance gain from the waveform diversity can also be understood by considering the additional phase center positions that result from a combination of the nonseparable radar pulse encoding on transmit with a multiaperture receiver system. Fig. 9 compares the effective phase center positions of a classical DPCA system (left) with the multidimensional waveform encoding technique (right). For the classical DPCA system using a single illuminator, one obtains for each transmitted pulse a total of $N_{\mathrm{Rx}}$ effective phase center positions that are spatially separated by a distance of $d_{\text {ant }} / 2$ from each other, where $d_{\text {ant }}$ is the distance between the Rx subaperture phase centers in the along-track direction. The maximum distance of the effective phase centers for one single Tx pulse is then given by

$$
d_{\max }=\frac{N_{\mathrm{Rx}}-1}{2} \cdot d_{\mathrm{ant}}
$$

where $N_{\mathrm{Rx}}$ is the number of subaperture elements. The use of multidimensional waveform encoding now leads to additional phase centers between each $\mathrm{Tx} / \mathrm{Rx}$ aperture pair. If we assume the same number $N=N_{\mathrm{Tx}}=N_{\mathrm{Rx}}$ and equal positions $x_{i, \mathrm{Tx}}=x_{i, \mathrm{Rx}}$ for the $\mathrm{Tx}$ and $\mathrm{Rx}$ apertures, we obtain a total of $2 N-1$ independent phase center positions that span a total length of

$$
d_{\max }=(N-1) \cdot d_{\text {ant }} .
$$

This length is twice the length of the classical DPCA system that employs a single transmitter. The additional phase centers provide for each transmitted pulse an increased number of azimuth samples along the synthetic aperture, which allows for improved ambiguity suppression in the case of pointlike targets. This bears, in turn, the potential to reduce either the PRF or the overall antenna length by a factor of two. Another opportunity is an enhanced detection and parameter estimation performance in a multibaseline along-track interferometer and/or ground moving target indication system due to the increased length of the total along-track baseline.

At first glance, one may believe that orthogonal Tx waveforms are also well suited to reduce azimuth ambiguities in a multiaperture high-resolution wide-swath SAR imaging system. However, the mere use of simultaneously transmitted orthogonal waveforms will only disperse - but not suppress - the ambiguous energy, thereby making this approach suitable only for the attenuation of ambiguous returns from pointlike targets in specialized scenarios (see also [50] and [51]). Suppression of ambiguous returns from distributed targets can, nevertheless, be obtained by a slight modification of the previous multiaperture $\mathrm{Tx} / \mathrm{Rx}$ system. The basic idea behind this modification is to combine the spatial transmit diversity in azimuth with digital beamforming on receive in elevation. For this, the signals from the individual azimuth apertures are no longer transmitted simultaneously but in sequence by dividing the total Tx pulse, again, into multiple subpulses, where the number of subpulses corresponds to the number of azimuth apertures. The scattered signals from the different subpulses will then-at each instant of time-arrive from different elevation angles, and it becomes possible to separate the radar echoes from the different subpulses by digital beamforming on receive in elevation. Hence, this spatial filtering will not only disperse, but also suppress, the ambiguous energy from distributed scatterers.

An alternative to the sequential transmission from multiple azimuth apertures is the formation of multiple narrow azimuth beams in the transmitter, thereby reducing the Doppler bandwidth in the receiver channels, as schematically shown on the right part of Fig. 6. This solution has the advantage of always using all Tx antenna elements, which alleviates the peak power requirements of the $T / R$ modules to achieve a predefined SNR. A sequence of chirp signals is then transmitted while switching between different azimuth beams from subpulse to subpulse. Fig. 10 shows a schematic example for such multidimensional waveform encoding for three chirped subpulses as a function of range time $\tau$ and azimuth angle $\theta$. This specific illumination sequence results for each point on the ground during the formation of the synthetic aperture 


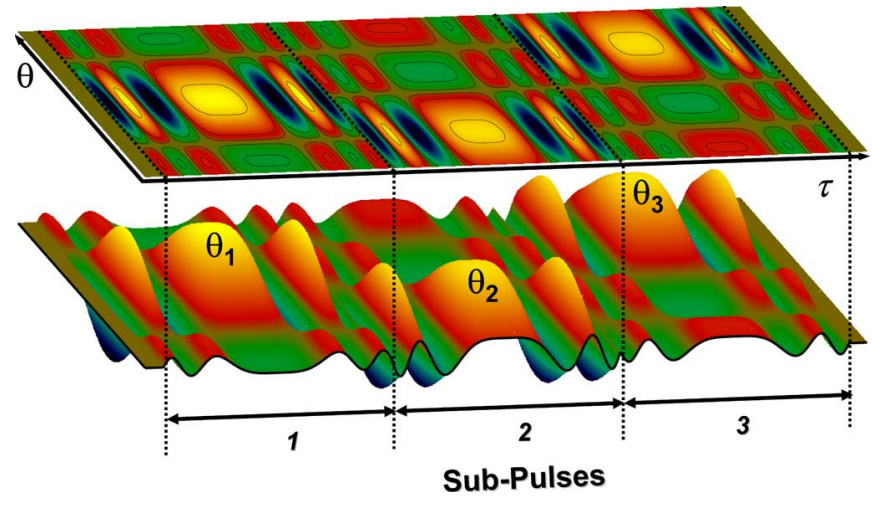

Fig. 10. Multidimensional radar waveform encoding in range time $(\tau)$ and azimuth $(\theta)$. The figure shows the transmitted pulse that consists of three successive range chirps indicated by the numbers 1 to 3 . Each subpulse is steered to a different azimuth angle $\theta$ by using an appropriate linear phase ramp for the antenna excitation coefficients. The azimuth antenna pattern for each narrow subbeam has been approximated by a $\sin (x) / x$ function, where the beamwidth corresponds to the overall length of the Tx antenna array.

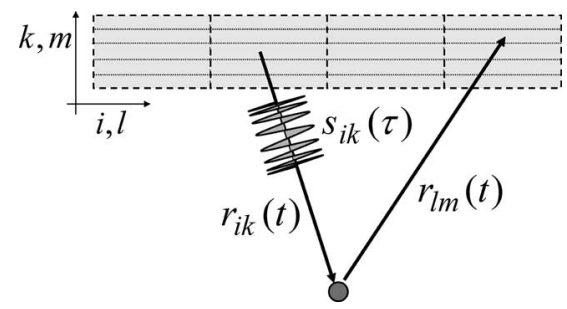

Fig. 11. Signal model for the response of a multiaperture $\mathrm{T} x / \mathrm{Rx}$ system to an isolated point scatterer.

in multiple and mutually delayed chirp signal returns. If we consider now a scatterer at a given range, one will, at each instance of time, only receive the scattered signal from one subpulse while the other subpulses lead to a superposition of the received signal with echoes from scatterers located at different azimuth positions and ranges. The different ranges are, in turn, associated with different look angles in elevation. It is hence possible to suppress the simultaneously received returns from different ranges by digital beamforming on receive in elevation, which enables a clear and unambiguous separation of the radar echoes from the different azimuth beams. The echoes from multiple azimuth beams can finally be combined coherently to recover the full Doppler spectrum for a high azimuth resolution. This combination is equivalent to a signal reconstruction from a multichannel bandpass decomposition, where the individual bandpass signals correspond to narrowband azimuth spectra with different Doppler centroids.

\section{B. Point Target Model}

For a mathematical description, we consider the signal reception of a multiaperture Tx/Rx SAR system in response to an isolated point scatterer, as shown in Fig. 11. For convenience, we consider all signals to be in the baseband and further neglect any constant factors reflecting free space attenuation of the electromagnetic waves, system losses, as well as the constant radar cross section of the point scatterer. The signal $u_{l m}(\tau, t)$ received by the subaperture element of column $l$ and row $m$ is then given by the linear superposition of the echoes from all transmit signals as follows:

$$
u_{l m}(\tau, t)=\sum_{i, k}^{N_{\text {Tx }}} s_{i k}\left(\tau-\frac{r_{i k l m}(t)}{c_{0}}\right) \cdot \exp \left(-j \frac{2 \pi}{\lambda} r_{i k l m}(t)\right)
$$

where $s_{i k}(\tau)$ denotes the amplitude- and phase-modulated signal transmitted by subaperture element $i k$. The variables $\tau$ and $t$ refer to fast (i.e., range) and slow (i.e., azimuth) times, respectively. Note that each of the transmitted signals $s_{i k}(\tau)$ consists, in general, of multiple subpulses with different phase coefficients to accomplish the desired beamsteering in azimuth within one complete pulse. In (6), the velocity of electromagnetic wave propagation in free space and the wavelength are represented by $c_{0}$ and $\lambda$, respectively, and the function $r_{i k l m}(t)$ describes the range history for each $\mathrm{Tx} / \mathrm{Rx}$ subarray pair, which is defined as

$$
r_{i k l m}(t)=r_{i k}(t)+r_{l m}(t)
$$

where $r_{i k}(t)$ is the range from the transmit antenna element $i k$ to the point scatterer, and $r_{l m}(t)$ is the range from the point scatterer to the receiving aperture element with index $l \mathrm{~m}$.

The nonseparable waveform encoding, as exemplified by Fig. 10, now causes systematic differences in the phase and amplitude of the returned echoes, depending on the azimuth direction $\theta$. This can be taken into account during range focusing by using, for each azimuth angle $\theta$, a different matched filter for range compression. Range compression is hence (in general) no longer independently performed for the $n_{\mathrm{Rx}}$ receive apertures but uses a manifold of matched filters applied to weighted superpositions of signals from the Rx antenna elements. As a result, one obtains for each row $m$ of the Rx array the following 3-D range compressed signal:

$$
\begin{aligned}
& =\left\{\sum_{l=1}^{w_{l}}(\tau, t, \theta)\right. \\
& \otimes\left\{\sum_{i, k}^{N_{\mathrm{Tx}}} s_{i k}^{*}(-\tau) \cdot \exp \left(-j \frac{2 \pi}{\lambda}\left(i-\frac{N_{i}+1}{2}\right) d_{\mathrm{ant}} \sin (\theta)\right)\right\}
\end{aligned}
$$

where the first sum on the right-hand side of (8) describes the formation of a narrow $\mathrm{Rx}$ beam in the direction of $\theta$ by a weighted linear superposition of the Rx signals in one row of the antenna array, and the second sum is the matched filter for range focusing, corresponding to the expected "replica" from that direction. As mentioned before, the distance between the subapertures in azimuth is indicated by $d_{\text {ant }}$ which implicitly 

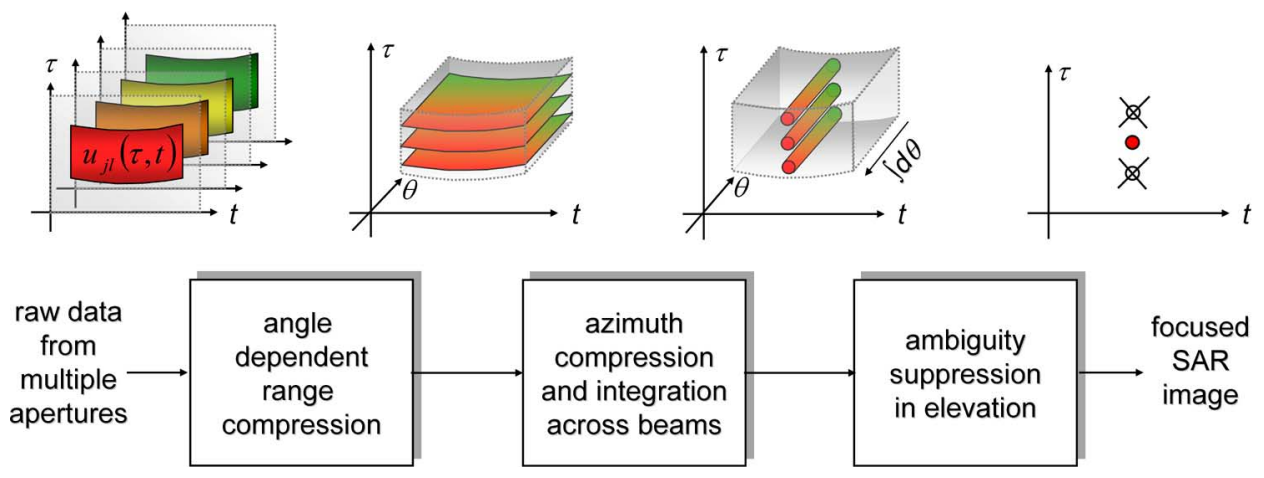

Fig. 12. Main processing steps for multiaperture SAR focusing in systems that employ multidimensional waveform encoding.

assumes an equal subaperture spacing for the transmit and receive chains. ${ }^{7}$

Azimuth compression can now be accomplished by using a matched azimuth filter for each $\theta$ separately and a subsequent integration across all azimuth beams $\theta$. Hence, we have

$$
a_{m}(\tau, t)=\int_{\theta_{\min }}^{\theta_{\max }}\left[w_{m}(\tau, t, \theta) \otimes \exp \left(j \frac{2 \pi}{\lambda} r_{m}(t)\right)\right] \cdot d \theta
$$

where

$$
r_{m}(t)=\frac{1}{N_{\mathrm{Tx}} N_{l}} \sum_{i, k}^{N_{\mathrm{Tx}}} \sum_{l=1}^{N_{l}} r_{i k l m}(t)
$$

is the range history within each beam. The integration corresponds to the aforementioned reconstruction of the full bandwidth signal from multiple Doppler subbands. In practice, the integration is substituted by a summation, and the upper and lower limits $\theta_{\min }$ and $\theta_{\max }$ determine the overall Doppler bandwidth after the reconstruction of the whole azimuth spectrum from its bandpass decomposition. The resulting SAR images $a_{m}(\tau, t)$ are now well focused in terms of azimuth, but still ambiguous in terms of range.

The remaining range ambiguities may now be suppressed by combining the SAR images from the $N_{m}$ antenna rows via digital beamforming on receive in elevation, i.e.,

$$
p(\tau, t)=\sum_{m=1}^{N_{m}} h_{m}(\tau) \cdot a_{m}(\tau, t)
$$

\footnotetext{
${ }^{7}$ This derivation assumes a short Tx pulse and neglects the movement of the platform during pulse transmission. In the case of long Tx pulses, one may avoid such a start-stop approximation by introducing an additional phase function within the second sum of (8). This phase function, which depends on the angle $\theta$, compensates for the systematic phase shift between the echoes from the different subpulses. Another opportunity to incorporate such a time delay is a systematic modification of the Tx and Rx array positions for each subpulse. For convenience, we will further neglect range migration in the derivation of the following formulas, which facilitates the understanding of the essential processing steps.
}

such that

$$
h_{m}(\tau): \begin{cases}\sum_{m=1}^{N_{m}} h_{m}(\tau)=\max , & \text { for } \theta_{\mathrm{el}}(\tau) \\ \sum_{m=1}^{N_{m}} h_{m}(\tau)=\min , & \text { for } \theta_{\mathrm{el}, \mathrm{amb}}(\tau)\end{cases}
$$

where $\theta_{\text {el,amb }}$ denotes the elevation angles with ambiguous returns. In practice, it is not necessary to minimize the range ambiguities by space-variant null steering (which requires also an accurate digital elevation model), but it would be sufficient to shape the Rx elevation beam such that the range ambiguities remain below a given level of, e.g., $-25 \mathrm{~dB}$. The illustrations in Fig. 12 summarize the main processing steps.

\section{System Design Example}

To illustrate the achievable performance gain from such multidimensional waveform encoding in azimuth, we consider the design of a spaceborne X-band SAR system with an azimuth resolution of $1.5 \mathrm{~m}$ and a swath width of $135 \mathrm{~km}$. Such a system enables a "continuous" high-resolution observation of the total Earth's surface with a 20-day repeat cycle at a mean satellite altitude of $576 \mathrm{~km}$. A quasi-continuous observation of the Earth's surface is, e.g., desired for differential and permanent scatterer interferometry. The intended swath width limits the maximum possible PRF by

$$
\mathrm{PRF}<\left[\frac{2 \cdot\left(r_{\mathrm{far}}-r_{\text {near }}\right)}{c_{0}}+2 \cdot n_{\mathrm{sub}} \cdot \tau_{\mathrm{p}}\right]^{-1}
$$

where $\tau_{\mathrm{p}}$ is the pulse duration of each subpulse, $n_{\mathrm{sub}}$ is the number of adjacent subpulses, and $r_{\text {far }}$ and $r_{\text {near }}$ are the slant range distances in the far and near ranges, respectively. Further restrictions result from the timing diagram, as shown in Fig. $13 .^{8}$

In the following, we assume a minimum incident angle of $27^{\circ}$, which corresponds to a near range $r_{\text {near }}=639.6 \mathrm{~km}$. For a $135-\mathrm{km}$ swath, the maximum incident angle is then $37.9^{\circ}$,

\footnotetext{
${ }^{8}$ This analysis is based on a spherical Earth model with $r_{\mathrm{E}}=6370 \mathrm{~km}$. It is further assumed that nadir echoes can be suppressed to a sufficiently low level by digital beamforming on receive in elevation. Otherwise, one would have to select a different incident angle range. Such a final optimization is beyond the scope of this paper.
} 


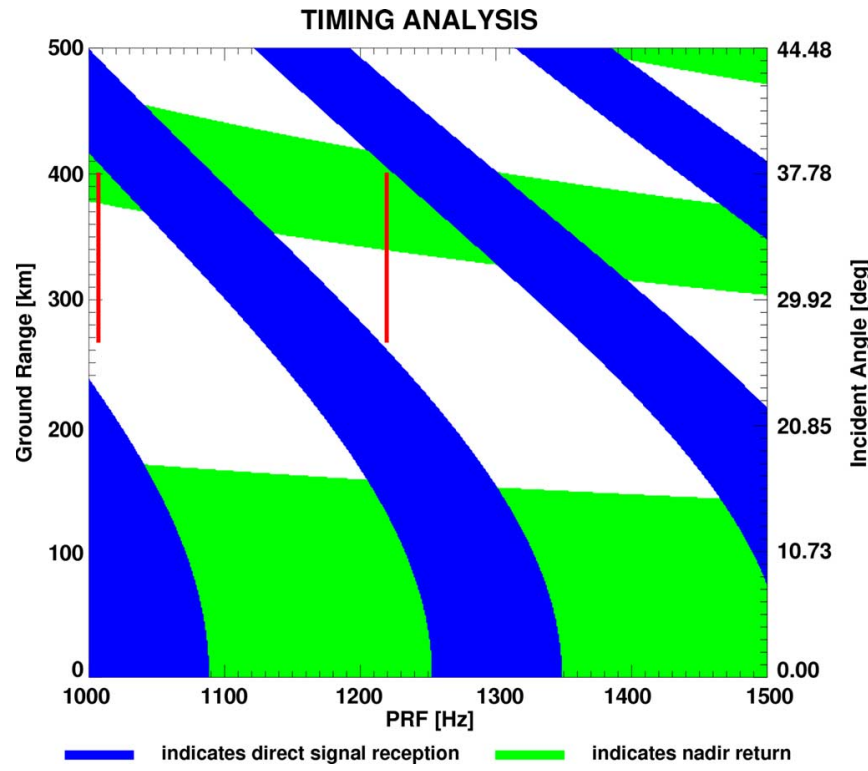

Fig. 13. Timing diagram for the exemplary SAR system.

corresponding to a far range $r_{\text {far }}=712.5 \mathrm{~km}$. As shown in Fig. 13, a set of possible PRFs would then range from 1215 to $1224 \mathrm{~Hz}$. As can be seen in (13), these PRFs limit, in turn, the maximum pulse duration to $\tau_{\mathrm{p}} \leq 55 \mu \mathrm{s}$, assuming a transmit pulse with $n_{\text {sub }}=3$ azimuth beams. In the following, we chose a PRF of $1220 \mathrm{~Hz}$ and limit the subpulse duration to $\tau_{\mathrm{p}} \leq 50 \mu \mathrm{s}$, which gives us some guard time, as well as some additional margin for switching the beams between the subpulses. To resolve the range ambiguities from multiple subpulses, the antenna must then have a total height $h_{\text {ant }}$ of at least ${ }^{9}$

$$
h_{\mathrm{ant}} \geq \frac{2 \lambda \cdot r_{\mathrm{far}} \cdot \tan \left(\theta_{\text {inc }, \max }\right)}{c_{0} \cdot \tau_{\mathrm{p}}}
$$

which ensures a reliable separation between the multiple radar echoes by digital beamforming on receive. In this example, the height of an optimally oriented antenna should be larger than $\sim 2.3 \mathrm{~m}$. Table I summarizes the selected system parameters.

Fig. 14 compares the azimuth ambiguities for different SAR system configurations. The results have been obtained from a point target simulation using the processing steps outlined in (8)-(12) and the parameters of Table I. The column on the left shows the response of a classical SAR system. The image on the upper left illustrates the system configuration that consists of a single $2.5-\mathrm{m} \mathrm{Tx} / \mathrm{Rx}$ aperture. The 3-D surface plot in the middle left shows the magnitude of the processed SAR image as a function of range and azimuth, assuming a PRF of $1220 \mathrm{~Hz}$. It becomes clear that the low PRF causes severe azimuth ambiguities, which are further quantified in azimuth cut shown on the lower left. The first azimuth ambiguities have an almost comparable level to the main response, which is shown in the center of the plot at an azimuth position of $0 \mathrm{~m}$.

\footnotetext{
${ }^{9}$ This equation has been obtained from the assumption that an optimally oriented antenna should be large enough to direct a beam with a maximum gain toward the desired ground range position and to simultaneously suppress range ambiguities from adjacent subpulses by nulls in the antenna pattern. In practice, one may relax this equation by tolerating a given ambiguity level and/or a slightly lower Rx antenna gain.
}

The second column in Fig. 14 illustrates the performance improvement that can be gained by using three additional Rxonly apertures for signal reception in azimuth. This case corresponds to the ambiguity suppression for the HRWS system. The additional samples along the synthetic aperture decrease the major azimuth ambiguities to a magnitude of $-17 \mathrm{~dB}$. A further improvement can be obtained by using the multidimensional waveform encoding in azimuth. For this, the Tx pulse is subdivided into three subpulses, as shown in the third column of Fig. 14 on the top. Each of the subpulses is transmitted with a different azimuth (or squint) angle by switching between three linear phase ramps in the antenna excitation pattern. As shown in the middle image, this improves the azimuth ambiguity suppression at the cost of additional range ambiguities. The ambiguous azimuth energy is hence transformed to a different range time, which corresponds to a different elevation angle. The range ambiguities are now suppressed by digital beamforming on receive in elevation, as shown in the illustration on the upper right. The remaining azimuth ambiguities have a magnitude of $-30 \mathrm{~dB}$, as shown in third column on the bottom. Finally, the inset on the lower right shows a zoom of the 2-D SAR impulse response in the range of $\pm 10 \mathrm{~m}$. The azimuth resolution is approximately $1.5 \mathrm{~m}$, which is in good agreement with the predicted resolution for the processed Doppler bandwidth of $5000 \mathrm{~Hz}^{10}$

This performance investigation demonstrates that it becomes possible to design compact SAR sensors with high resolutions and wide coverage by employing the principle of multidimensional waveform encoding. As shown in the exemplary analysis, an X-band SAR system with an azimuth resolution ${ }^{11}$ of $1.5 \mathrm{~m}$, covering a swath width of $135 \mathrm{~km}$, can be built with a compact antenna of approximately $10 \mathrm{~m}$ in length and $2.5 \mathrm{~m}$ in height onboard a LEO satellite that orbits at a mean altitude of $576 \mathrm{~km}$. For comparison, TerraSAR-X, which orbits at a slightly lower altitude of $514 \mathrm{~km}$, has in stripmap mode an azimuth resolution of approximately $3 \mathrm{~m}$ and a nominal swath width of $30 \mathrm{~km}$ [12]. The performance improvement in our example becomes possible by the systematic combination of two complementary digital radar techniques, namely, multiaperture recording on receive and multidimensional waveform encoding on transmit. Further improvements are possible, e.g., by increasing the number of subpulses and/or the number of subaperture elements. In the case of the previously mentioned sequential excitation of the azimuth subapertures, it is, moreover, possible to optimize

\footnotetext{
${ }^{10}$ Note that the processed Doppler bandwidth $B_{\text {proc }}=5000 \mathrm{~Hz}$ exceeds, in this example, $N_{\mathrm{Rx}} \cdot \mathrm{PRF}=4880 \mathrm{~Hz}$. This is in accordance with the reconstruction from the $3-\mathrm{D}$ range compressed signal in (9), which integrates across multiple narrowband azimuth "looks," formed by the multiplicative superposition of the Tx and Rx azimuth antenna patterns. At first glance, one may regard the fact that the processed bandwidth exceeds the number of azimuth samples (i.e., $B_{\text {proc }}>N_{\mathrm{Rx}} \cdot \mathrm{PRF}$ ) as a fundamental contradiction to the generalized Nyquist sampling theorem. However, this contradiction is immediately resolved if one takes into account that the additional information is not stored in the azimuth but in the elevation plane of the recorded radar data volume. Multidimensional waveform encoding in azimuth is hence another means of reducing the redundancy within large receiver arrays.

${ }^{11}$ The azimuth resolution does not depend on the antenna length but on the size of the subaperture elements. By increasing the number of subapertures and keeping the whole antenna length fixed, it would, hence, be possible to further improve the resolution.
} 
TABLE I

PARAMETERS USED IN THE SyStem Simulation

\begin{tabular}{|l|l|l|l|}
\hline Parameter & Value & Parameter & Value \\
\hline Swath Width & $135 \mathrm{~km}$ & Number of Sub-Pulses & 3 \\
\hline Orbital Altitude & $576 \mathrm{~km}$ & Sub-Pulse Duration & $50 \mu \mathrm{s}$ \\
\hline Repeat Cycle & 20 days & PRF & $1220 \mathrm{~Hz}$ \\
\hline Repeat Orbits & 299 & Azimuth Sub-Apertures & 4 \\
\hline Incident Angle Range & $27.0^{\circ}-37.9^{\circ}$ & Antenna Length & $4 \times 2.5 \mathrm{~m}$ \\
\hline Wavelength & $0.031 \mathrm{~m}$ & Antenna Height & $2.5 \mathrm{~m}$ \\
\hline Bandwidth & $200 \mathrm{MHz}$ & Processed Azimuth Bandwidth & $5000 \mathrm{~Hz}$ \\
\hline Ground Range Resolution & $1.5 \mathrm{~m}\left(30^{\circ}\right)$ & Azimuth Resolution & $1.5 \mathrm{~m}$ \\
\hline
\end{tabular}
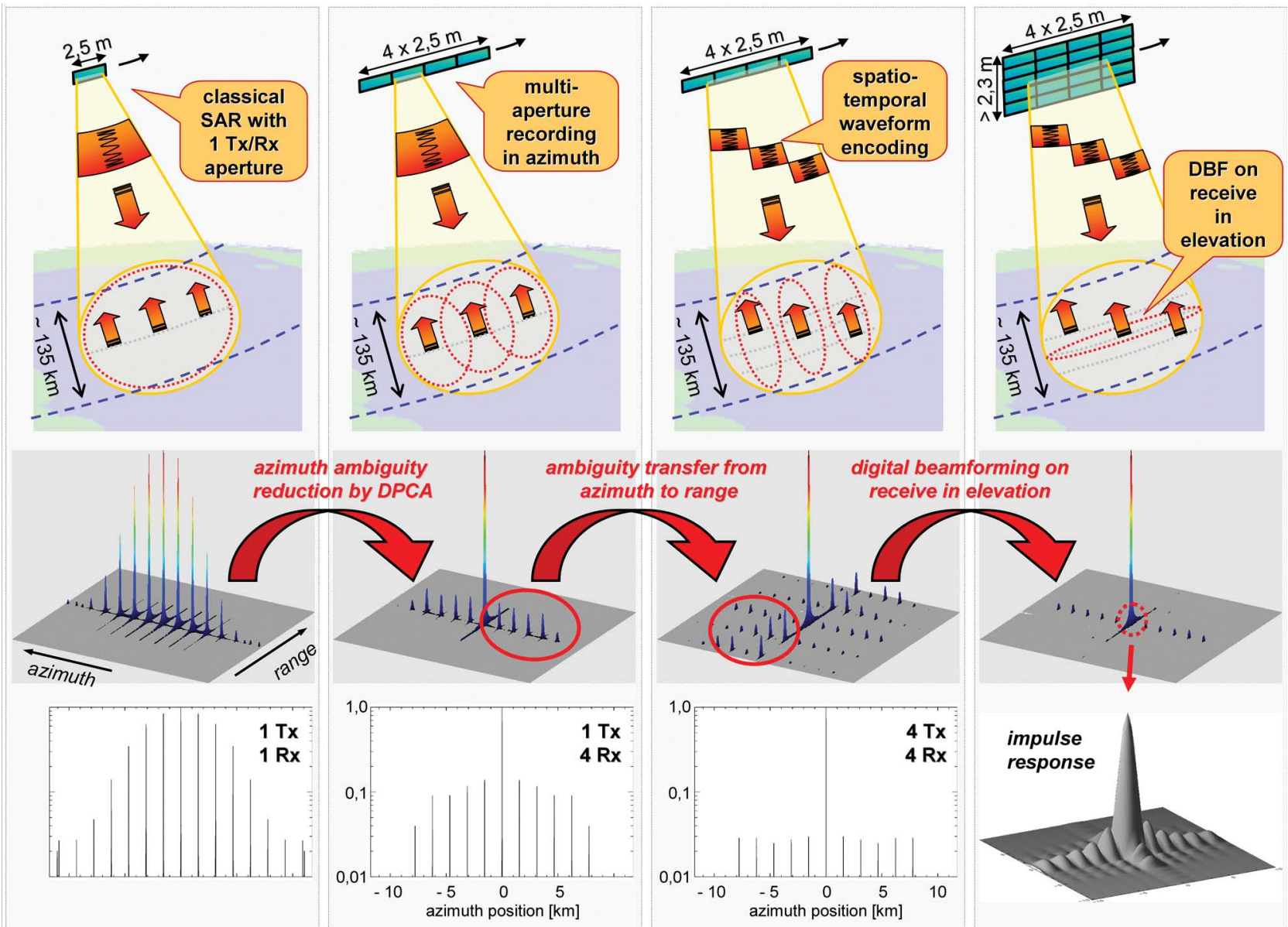

\section{digital beamforming on} receive in elevation
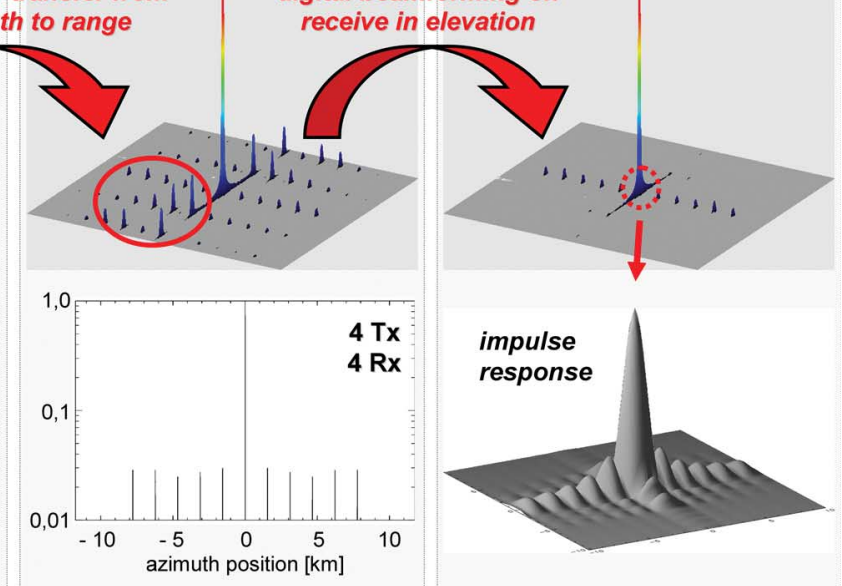

Fig. 14. Transfer of ambiguous energy from azimuth to range by nonseparable radar waveform encoding for four transmit and four receive channels. (Left) Classical SAR with one transmitter and one receiver aperture. (Middle left) Azimuth ambiguity suppression by a classical DPCA system with four independent receive apertures. (Middle right) Ambiguity transfer from azimuth to range by intrapulse azimuth beamsteering with three subpulses. (Right) Range ambiguity suppression by digital beamforming on receive in elevation. The top row illustrates the aperture arrangement, the transmitted waveform, and the spatial location of simultaneous returns from the ground; the middle row shows the processed SAR image from a single pointlike scatterer in range and azimuth; and the bottom row shows the magnitude of the azimuth ambiguities obtained from a slice through the processed SAR image. The shaded surface plot on the lower right is a 2-D zoom of the SAR impulse response.

the azimuth processing by explicitly taking into account the nonuniform sampling of the synthetic aperture [30], [33]. If compared to the DPCA and HRWS systems, which employ solely multiple-aperture recording on receive, this will allow for a reduction of the overall antenna length by a factor of up to two (cf., Fig. 9). This can then be used to build more 
compact antennas for easier spacecraft accommodation and/or to increase the unambiguous swath width.

The azimuth ambiguity suppression technique may further be combined with additional waveform encoding in elevation. Such a 3-D nonseparable time-azimuth-elevation encoding allows for the use of the complete antenna array for the transmission of all subpulses, thereby reducing the peak power requirements in the $\mathrm{T} / \mathrm{R}$ modules. A simple solution for a wide-swath illumination is by merely splitting the total transmitted range frequency band into multiple independent subbands, which are then assigned to different rows of the Tx antenna array. Another solution is an explicit combination of the azimuth encoding with the principle of intrapulse beamsteering in elevation, as introduced in Section IV. Further optimizations could employ an explicit informationtheoretic analysis of the remaining redundancies within the multidimensional signal space recorded by the total antenna array. Such an analysis is well suited to optimize the system performance and simultaneously minimize the redundancies in the stored multidimensional data volume. The redundancy reduction could either be limited to an optimization of the transmitted waveform, or one could, in addition, take advantage of the redundancy reduction techniques outlined in Section II-C. The systematic combination of both techniques is expected to be able to reduce the redundancies in the recorded data volume to a sufficiently low level without having to refer to sophisticated onboard processing. The systematic derivation of optimized spatiotemporal waveforms, which will, in general, employ more than two nonseparable dimensions, is beyond the scope of this paper, which serves the only purpose to introduce the basic principle of multidimensional waveform encoding and to illustrate its manifold potentials for the design and operation of future SAR systems.

\section{DISCUSSION}

The systematic combination of spatiotemporal radar waveform encoding on transmit with multiaperture digital beamforming on receive is an innovative concept that enables new and very powerful SAR imaging modes for a wide range of remote sensing applications. Examples are an improved SAR system performance by increasing the number of effective phase centers, larger along-track baselines for along-track interferometry and moving object indication, and an efficient reduction of redundant information recorded by large receiver arrays. The opportunity to transfer ambiguous signal energy from azimuth to range via multibeam switching during each transmitted radar pulse further enables efficient suppression of azimuth ambiguities by spatiotemporal filtering of the recorded multiaperture data in elevation. From these examples, it becomes clear that the multidimensional nonseparable transmit waveform encoding has a high potential to significantly improve the performance of future SAR sensors and missions.

Digital beamforming on transmit further allows a flexible distribution of the RF signal energy on the ground. Not only does this enable a versatile switching between different imaging modes like multi-beam ScanSAR, spotlight, and HRWS stripmap, but it also allows for the simultaneous combination

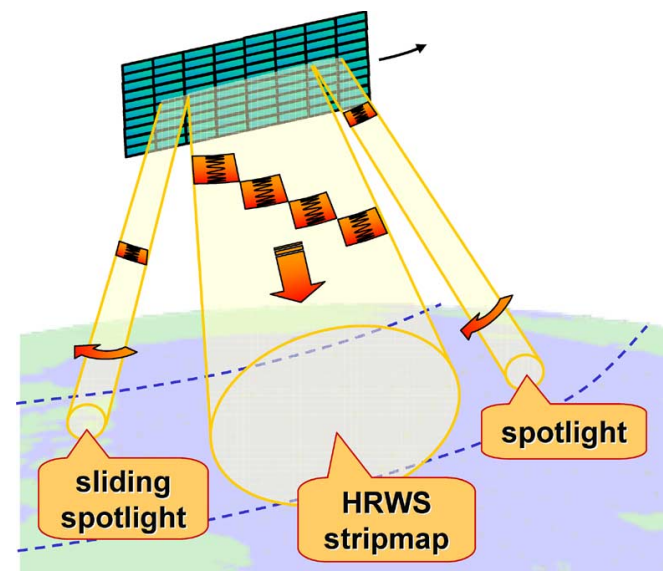

Fig. 15. Hybrid operation by multidimensional waveform encoding.

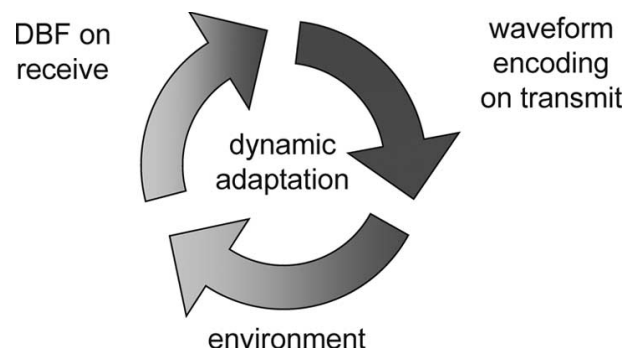

Fig. 16. Dynamic adaptation of the waveform encoding to the environment by closing the loop between the receiver and the transmitter.

of multiple imaging modes in one and the same satellite pass. An example is high-resolution spotlight-like mapping of an area of high interest in combination with wide-swath mapping for interferometric applications. This can be achieved by enhancing the multidimensional waveform encoding with additional subpulses that steer highly directive transmit beams to some specific areas on the ground. By this, one may achieve a high Tx gain and a longer illumination time along the synthetic aperture, which will improve both the radiometric and the geometric resolutions for a given area of interest. This is illustrated in Fig. 15, and such a hybrid mode will be well suited to satisfy otherwise contradicting user requirements like, for example, the conflict between a continuous interferometric background mission and a high-resolution data acquisition request.

The data acquisition in such a system could even be made adaptive where more system resources are automatically devoted to areas of high interest and/or low SNR, thereby maximizing the overall information content for a given RF power budget. By this, a closed loop will be formed, which directly connects the recorded data to the transmitted waveform by an appropriate real-time raw data evaluation of the scattered signals from the environment (cf., Fig. 16). As a simple opportunity, one may consider the adaptive use of a longer subpulse and/or a higher Tx antenna gain for a spatially restricted area with lower backscatter, thereby enhancing the overall SNR for a given amount of system resources. Such an adaptation can, for example, be performed in real time via a multiple beamforming processor that evaluates the spatial power distribution of the scattered and recorded radar data. The output from this processor is then directly fed back to the waveform encoding system. 
Another opportunity is the use of longer azimuth illumination times and higher range bandwidths for selected regions with high contrasts and/or fast changes. Such features could be indicators for areas of higher interest, therefore calling also for a higher spatial and/or radiometric resolution. A potential application scenario is, for example, operational ship detection in the open sea, which requires the frequent scanning of wide areas. In this scenario, the recorded multiaperture signals may now be evaluated onboard the satellite by a suboptimum realtime moving target indicator (MTI) feature detector with low complexity. In case of some evidence for a moving object or ship in one area of the scene, one may illuminate that region with a highly directive subbeam to improve both the spatial resolution and the MTI performance without loosing the general overview about the residual scene. The information gain from long observation times is also well known from high-resolution airborne radar data [47]. Such an adaptive SAR system can then be regarded as a first step toward a cognitive radar that directs its resources to areas of high interest in analogy to the selective attention mechanisms of the human visual system with its saccadic eye movements [52], [53]. The rising interest in such systems with regard to a wide range of other radar applications is also documented in the recent literature [54], [55].

The technique of multidimensional waveform encoding, moreover, bears some interesting opportunities in conjunction with multistatic SAR systems [27], [34]. An example is the distribution of subpulses on multiple satellites, which not only allows for optimized system performance and coverage but also enables efficient phase synchronization between the involved radar instruments. This is of special importance for interferometric applications [56], [57]. The synchronization can be achieved without additional hardware via the common illuminated footprint by a slight modification of the "alternating" bistatic SAR mode [58]. In contrast to the original proposal where the transmitter is switched from pulse to pulse, there will be no rise of azimuth ambiguities.

The great flexibility in operating digital radar systems with multidimensional waveform encoding on transmit and digital beamforming on receive requires, of course, new strategies to optimize the overall system performance. The derivation of optimized system designs and waveforms may also incorporate elements from Shannon's information theory [59], [60] like, for example, maximization of the mutual information between the scene parameters and the recorded radar signals for a given RF power budget. Such an optimization bears several similarities to the questions arising about the optimum use of the channel capacity provided by MIMO communication systems. The information-theoretic optimizations may, moreover, help to get rid of the redundancies in the data recorded by multiaperture SAR systems. This is an essential requirement to cope with the omnipresent storage and data downlink bottlenecks.

\section{CONCLUSION}

From the examples in this paper, it has become clear that the combination of digital waveform encoding on transmit with digital beamforming on receive has the potential of significantly improving the capabilities and the performance of future
SAR systems and missions. The new generation of digital radar sensors will be able to resolve the hitherto contradicting requirements for high resolution, frequent monitoring, and wide coverage. Furthermore, hybrid and adaptive SAR imaging modes can be implemented by varying the pulse sequence in azimuth slow time. This additional degree of freedom is well suited for innovative operational concepts that satisfy a wider remote sensing user community with one and the same satellite mission. This will, in turn, increase the user acceptance and application range of SAR data, thereby justifying the necessary investments for the development and implementation of a new generation of more powerful SAR systems and missions. The analyses and suggestions presented in this paper should be regarded as a first step in this direction.

\section{ACKNOWLEDGMENT}

The authors would like to thank the anonymous reviewers for their valuable comments and suggestions to improve the readability of this paper.

\section{REFERENCES}

[1] F. Li and W. T. K. Johnson, "Ambiguities in spaceborne synthetic aperture radar systems," IEEE Trans. Aerosp. Electron. Syst., vol. AES-19, no. 3, pp. 389-397, May 1983.

[2] C. Elachi, Spaceborne Radar Remote Sensing: Applications and Techniques. New York: IEEE Press, 1988.

[3] J. C. Curlander and R. N. McDonough, Synthetic Aperture Radar: Systems and Signal Processing. New York: Wiley, 1991.

[4] A. Freeman, W. T. K. Johnson, B. Huneycutt, R. Jordan, S. Hensley, P. Siqueira, and J. Curlander, "The myth of the minimum SAR antenna area constraint," IEEE Trans. Geosci. Remote Sens., vol. 38, no. 1, pp. 320-324, Jan. 2000.

[5] K. Tomiyasu, "Conceptual performance of a satellite borne, wide swath synthetic aperture radar," IEEE Trans. Geosci. Remote Sens., vol. GRS-19, no. 2, pp. 108-116, Apr. 1981.

[6] R. K. Moore, J. P. Claassen, and Y. H. Lin, "Scanning spaceborne synthetic aperture radar with integrated radiometer," IEEE Trans. Aerosp. Electron. Syst., vol. AES-17, no. 3, pp. 410-421, May 1981.

[7] D. Massonnet, "Radar interferometry and its application to changes in the Earth's surface," Rev. Geophys., vol. 36, no. 4, pp. 441-499, 1998.

[8] A. Ferretti, C. Prati, and F. Rocca, "Nonlinear subsidence rate estimation using permanent scatterers in differential SAR interferometry," IEEE Trans. Geosci. Remote Sens., vol. 38, no. 5, pp. 2202-2212, Sep. 2000.

[9] M. Davidson, E. Attema, B. Rommen, N. Floury, L. M. Partricio, and G. Levrini, "ESA sentinel-1 SAR mission concept," in Proc. EUSAR, Dresden, Germany, 2006.

[10] F. De Zan and A. M. Monti Guarnieri, "TOPSAR: Terrain observation by progressive scans," IEEE Trans. Geosci. Remote Sens., vol. 44, no. 9, pp. 2352-2360, Sep. 2006.

[11] W. Carrara, R. Goodman, and R. Majewski, Spotlight Synthetic Aperture Radar: Signal Processing Algorithms. Boston, MA: Artech House, 1995.

[12] M. Stangl, R. Werninghaus, B. Schweizer, C. Fischer, M. Brandfass, J. Mittermayer, and H. Breit, "TerraSAR-X technologies and first results," Proc. Inst. Electr. Eng.-Radar, Sonar Navig., vol. 153, no. 2, pp. 86-95, Apr. 2006.

[13] L. C. Morena, K. V. James, and J. Beck, "An introduction to the RADARSAT-2 mission," Can. J. Remote Sens., vol. 30, no. 3, pp. 221234, Jun. 2004.

[14] L. Candela and F. Caltagrione, "Cosmo-SkyMed: Mission definition, main applications and products," in Proc. PolinSAR Workshop, ESA ESRIN, Frascati, Italy, 2003.

[15] [Online]. Available:http://www.ohb-system.de/Satellites/Missions/ sarlupe.html

[16] J. P. Claassen and J. Eckerman, "A system for wide swath constant incident angle coverage," in Proc. Synthetic Aperture Radar Technol. Conf., Las Cruces, NM, 1978.

[17] A. Jain, "Multibeam synthetic aperture radar for global oceanography," IEEE Trans. Antennas Propag., vol. AP-27, no. 4, pp. 535-538, Jul. 1979 
[18] B. R. Jean and J. W. Rouse, "A multiple beam synthetic aperture radar design concept for geoscience applications," IEEE Trans. Geosci. Remote Sens., vol. GRS-21, no. 2, pp. 201-207, Apr. 1983.

[19] H. Griffiths and P. Mancini, "Ambiguity suppression in SARs using adaptive array techniques," in Proc. IGARSS, Espoo, Finland, 1991, pp. 1015-1018.

[20] A. Currie and M. A. Brown, "Wide-swath SAR," Proc. Inst. Electr. Eng. F-Radar Signal Process., vol. 139, no. 2, pp. 122-135, Apr. 1992

[21] G. D. Callaghan and I. D. Longstaff, "Wide-swath space-borne SAR using a quad-element array," Proc. Inst. Electr. Eng.-Radar, Sonar Navig., vol. 146, no. 3, pp. 159-165, Jun. 1999.

[22] N. Goodman, D. Rajakrishna, and J. Stiles, "Wide swath, high resolution SAR using multiple receive apertures," in Proc. Int. Geosci. Remote Sens. Symp., Hamburg, Germany, 1999, vol. 3, pp. 1767-1769.

[23] M. Suess and W. Wiesbeck, "Side-looking synthetic aperture radar system," Eur. Patent EP 1241487 A 1, 2001.

[24] W. Wiesbeck, "SDRS: Software-defined radar sensors," in Proc. Int. Geosci. Remote Sens. Symp., Sydney, Australia, 2001, pp. 3259-3261.

[25] M. Suess, B. Grafmueller, and R. Zahn, "A novel high resolution, wide swath SAR system," in Proc. Int. Geosci. Remote Sens. Symp., Sydney, Australia, 2001, pp. 1013-1015.

[26] M. Suess, M. Zubler, and R. Zahn, "Performance investigation on the high resolution, wide swath SAR system," in Proc. EUSAR, Ulm, Germany, 2002, pp. 187-191.

[27] G. Krieger and A. Moreira, "Potentials of digital beamforming in bi- and multistatic SAR," in Proc. Int. Geosci. Remote Sens. Symp., Toulouse, France, 2003, pp. 527-529.

[28] C. Heer, F. Soualle, R. Zahn, and R. Reber, "Investigations on a new high resolution wide swath SAR concept," in Proc. Int. Geosci. Remote Sens. Symp., Toulouse, France, 2003, pp. 521-523.

[29] M. Younis, C. Fischer, and W. Wiesbeck, "Digital beamforming in SAR systems," IEEE Trans. Geosci. Remote Sens., vol. 41, no. 7, pp. 17351739, Jul. 2003.

[30] G. Krieger, N. Gebert, and A. Moreira, "Unambiguous SAR signal reconstruction from nonuniform displaced phase center sampling," IEEE Geosci. Remote Sens. Lett., vol. 1, no. 4, pp. 260-264, Oct. 2004.

[31] M. Younis, "Digital beam-forming for high-resolution wide swath real and synthetic aperture radar," Ph.D. dissertation, Univ. Karlsruhe, Karlsruhe, Germany, 2004.

[32] C. Fischer, C. Heer, G. Krieger, and R. Werninghaus, "A high resolution wide swath SAR," in Proc. Eur. Conf. Synthetic Aperture Radar, Dresden, Germany, 2006

[33] N. Gebert, G. Krieger, and A. Moreira, "High resolution wide swath SAR imaging with digital beamforming-Performance analysis, optimization and system design," in Proc. EUSAR, Dresden, Germany, 2006.

[34] G. Krieger and A. Moreira, "Spaceborne bi- and multistatic SAR: Potential and challenges," Proc. Inst. Electr. Eng.-Radar, Sonar Navig., vol. 153, no. 3, pp. 184-198, Jun. 2006.

[35] G. Krieger, N. Gebert, and A. Moreira, "Hochauflösendes SynthetikApertur-Seitensicht-Radarsystem mittels Digital Beamforming," German Patent Application DE 102006022 814.6, 2006.

[36] G. Krieger, N. Gebert, and A. Moreira, "Digital beamforming techniques for spaceborne radar remote sensing," in Proc. EUSAR, Dresden, Germany, 2006.

[37] G. Krieger, N. Gebert, and A. Moreira, "Digital beamforming and nonuniform displaced phase centre sampling in bi- and multistatic SAR," in Proc. EUSAR, 2004, pp. 563-566.

[38] S. Chiu and C. Gierull, "Multi-channel receiver concepts for RADARSAT-2 ground moving target indication," in Proc. EUSAR, Dresden, Germany, 2006.

[39] T. Berger, Rate Distortion Theory. Englewood Cliffs, NJ: Prentice-Hall, 1971.

[40] H. Fiedler, E. Boerner, J. Mittermayer, and G. Krieger, "Total zero Doppler steering-A new method for minimizing the Doppler centroid," IEEE Geosci. Remote Sens. Lett., vol. 2, no. 2, pp. 141-145, Apr. 2005.

[41] N. S. Jayant and P. Noll, Digital Coding of Waveforms. Englewood Cliffs, NJ: Prentice-Hall, 1984.

[42] G. M. Kautz, "Phase-only shaped beam synthesis via technique of approximated beam addition," IEEE Trans. Antennas Propag., vol. 47, no. 5, pp. 887-894, May 1999.

[43] A. Trastoy, F. Ares, and E. Moreno, "Phase-only control of antenna sum and shaped patterns through null perturbation," IEEE Antennas Propag. Mag., vol. 43, no. 6, pp. 45-54, Dec. 2001

[44] J. H. G. Ender, D. Cerutti-Maori, and W. Burger, "Radar antenna architectures and sampling strategies for space based moving target recognition," in Proc. Int. Geosci. Remote Sens. Symp., Seoul, Korea, 2005, pp. 2921-2924
[45] R. E. Carande, "Dual baseline and frequency along-track interferometry," in Proc. Int. Geosci. Remote Sens. Symp., Houston, TX, 1992, pp. $1585-1588$.

[46] R. Scheiber, "Along- and across-track single pass interferometry using the E-SAR system," in Proc. IGARSS, Seattle, WA, 1998, pp. 1097-1099.

[47] A. R. Brenner and J. H. G. Ender, "Demonstration of advanced reconnaissance techniques with the airborne SAR/GMTI sensor PAMIR," Proc. Inst. Electr. Eng.-Radar, Sonar Navig., vol. 153, no. 2, pp. 152-162, Apr. 2006.

[48] G. Krieger, J. Mittermayer, S. Buckreuss, M. Wendler, T. Sutor, F. Witte, and A. Moreira, "Sector imaging radar for enhanced vision," Aerosp. Sci. Technol., vol. 7, no. 2, pp. 147-158, Mar. 2003.

[49] J. Klare, A. R. Brenner, and J. H. G. Ender, "A new airborne radar for 3D imaging-Image formation using the ARTRINO principle," in Proc. EUSAR, Dresden, Germany, 2006.

[50] G. Krämer, "Suppression of ambiguities by phase coding," in Proc EUSAR, Cologne, Germany, 2002, pp. 97-100.

[51] J. Mittermayer and J. M. Martinez, "Analysis of range ambiguity suppression in SAR by up and down chirp modulation for point and distributed targets," in Proc. IGARSS, Toulouse, France, 2003, pp. 4077-4079.

[52] G. Krieger, I. Rentschler, G. Hauske, K. Schill, and C. Zetzsche, "Object and scene analysis by saccadic eye-movements: An investigation with higher-order statistics," Spat. Vis., vol. 13, no. 2/3, pp. 201-214, Nov. 2000.

[53] K. Schill, E. Umkehrer, S. Beinlich, G. Krieger, and C. Zetzsche, "Knowledge-based scene analysis with saccadic eye-movements," $J$ Electron. Imaging, vol. 10, no. 1, pp. 152-160, 2001.

[54] F. Gini, "Knowledge-based systems for adaptive radar," IEEE Signal Process. Mag., vol. 23, no. 1, pp. 14-17, Jan. 2006.

[55] S. Haykin, "Cognitive radar: A way of the future," IEEE Signal Process. Mag., vol. 23, no. 1, pp. 30-40, Jan. 2006.

[56] G. Krieger and M. Younis, "Impact of oscillator noise in bistatic and multistatic SAR," IEEE Geosci. Remote Sens. Lett., vol. 3, no. 3, pp. 424 428, Jul. 2006.

[57] G. Krieger and A. Moreira, "Multistatic SAR satellite formations: Potentials and challenges," in Proc. Int. Geosci. Remote Sens. Symp., Seoul, Korea, 2005, pp. 2680-2684

[58] G. Krieger, A. Moreira, H. Fiedler, I. Hajnsek, M. Younis, M. Werner, and M. Zink, "The TanDEM-X mission: A satellite formation for high resolution SAR interferometry," IEEE Trans. Geosci. Remote Sens., vol. 45 , no. 11, pp. 3317-3341, Nov. 2007.

[59] W. Weaver and C. E. Shannon, The Mathematical Theory of Communication. Urbana, IL: Univ. Illinois Press, 1949.

[60] T. M. Cover and J. M. Thomas, Elements of Information Theory. New York: Wiley, 2006.

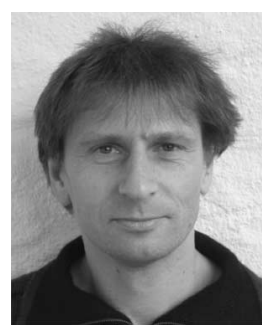

Gerhard Krieger (M'03) received the Dipl.Ing. (M.S.) and Dr.Ing. (Ph.D.) degrees (with honors) in electrical and communication engineering from the Technical University of Munich, Munich, Germany, in 1992 and 1999, respectively.

From 1992 to 1999 , he was with the LudwigMaximilians-University, Munich, where he worked as an interdisciplinary Research Scientist on the modeling of biological and technical vision systems. In 1999, he joined the Microwaves and Radar Institute (HR), German Aerospace Center (DLR), Wessling. Since 2001, he has been the Head of the New SAR Missions Group, DLR (HR). He is also currently Systems Engineer of the TanDEM-X mission. His research interests include the development of innovative remote sensing system concepts based on radar interferometry, tomography, bi- and multistatic satellite formations, digital beamforming, as well as advanced signal and image processing techniques. 


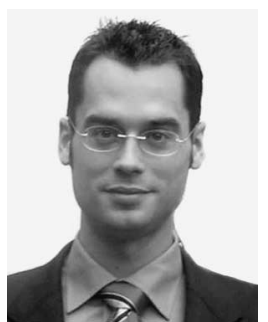

Nicolas Gebert received the Diploma degree from the Munich University of Technology, Munich, Germany, in 2003. He is currently working toward the Ph.D. degree at the Microwaves and Radar Institute (HR), German Aerospace Center (DLR), Wessling.

His current major research interests are signal processing, digital beamforming, system design, and optimization of multiaperture SAR systems for highperformance imaging applications.

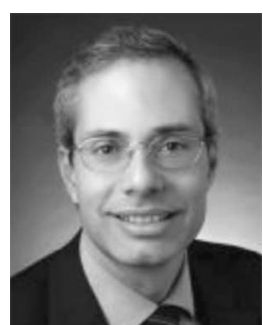

Alberto Moreira (M'92-SM'96-F'04) was born in São José dos Campos, Brazil, in 1962. He received the B.S. and M.S. degrees in electrical engineering from the Aeronautical Technological Institute, São José dos Campos, in 1984 and 1986, respectively, and the Eng.Dr. degree (honors) from the Technical University of Munich, Munich, Germany, in 1993.

In 2003, he received a Full Professorship from the University of Karlsruhe, Germany, in the field of microwave remote sensing. As its Chief Scientist and Engineer, he managed the SAR Technology Department, Microwaves and Radar Institute, German Aerospace Center (DLR) from 1996 to 2001. Under his leadership, the DLR airborne SAR system, which is called E-SAR, has been upgraded to operate in innovative imaging modes like polarimetric SAR interferometry and SAR tomography. Since 2001, he has been the Director of the Microwaves and Radar Institute, DLR, Oberpfaffenhofen, Germany. The institute contributes to several scientific programs and space projects for actual and future air- and spaceborne SAR missions. Recently, the mission proposal TanDEM-X that is led by his institute has been approved for the realization phase. He is the Principal Investigator for this mission. His professional interests and research areas encompass radar end-to-end system design and analysis, innovative microwave techniques and system concepts, signal processing, and remote sensing applications.

Prof. Moreira is a member of the IEEE GRSS Administrative Committee (1999-2001 and 2004-today), is the Chair of the German Chapter of the GRSS since 2003, and is actively serving as an Associate Editor for the IEEE Geoscience And Remote Sensing Letters. Since 2003, he has been also a member of the Board of Directors of the Information Technology Society of German Association for Electrical, Electronic, and Information Technologies. He has been contributing to the successful series of the European SAR conferences since 1996 as a member of the Technical Program Committee, the Technical Chairman (2000), the Awards Chairman (2002-2004), and the General Chairman (2006). In 1995, he was the recipient of the DLR Science Award. He and his colleagues received the GRSS Transactions Prize Paper Awards in 1997 and 2001, respectively. He was also the recipient of the IEEE Nathanson Award (1999) and the IEEE Kiyo Tomiyasu Award (2007). 